Supporting Information

For

\title{
A Stereoselective and Atom-Efficient Approach to Multifunctionalized Five and Six Membered Rings via a Novel Michael-Initiated Intramolecular Diels-Alder Furan Reaction
}

\author{
Irishi N.N. Namboothiri,* Madhu Ganesh, Shaikh M. Mobin, Miriam Cojocaru
}

\section{Table of Contents}

. Table S1. ${ }^{1} \mathrm{H}$ NMR data of 7a

Page

2. Table S2. ${ }^{1}$ H NMR data of $\mathbf{8 a}$

S-3

3. Table S3. ${ }^{1} \mathrm{H}$ NMR and 2D-NOESY data of $\mathbf{7 b}$

S-4

4. Table S4. ${ }^{1} \mathrm{H}$ NMR and 2D-NOESY data of $\mathbf{8 b}$

5. Table S5. ${ }^{1} \mathrm{H}$ NMR data of $\mathbf{7 d}$

S-6

6. Table S6. ${ }^{1} \mathrm{H}$ NMR data of $\mathbf{8 d}$

S-7

7. Table S7. ${ }^{1} \mathrm{H}$ NMR data of $\mathbf{7 e}$

S-8

8. Table S8. ${ }^{13} \mathrm{C}$ NMR data of 7a-e and 8a-e

S-9

9. Table S9. ${ }^{13} \mathrm{C}$ NMR data of $7 \mathbf{f}$ and $\mathbf{7 g}$

S-10

10. Figure $S 1 .{ }^{1} \mathrm{H}$ NMR spectrum of $\mathbf{6 a}$

S-10

11. Figure S2. ${ }^{13} \mathrm{C}$ NMR spectrum of $\mathbf{6 a}$

S-11

12. Figure S3. ${ }^{1} \mathrm{H}$ NMR spectrum of $\mathbf{6 b}$

S-12

13. Figure S4. ${ }^{13} \mathrm{C}$ NMR spectrum of $\mathbf{6 b}$

S-13

14. Figure S5. ${ }^{1} \mathrm{H}$ NMR spectrum of $\mathbf{6 c}$

S-14

15. Figure S6. ${ }^{13} \mathrm{C}$ NMR spectrum of $\mathbf{6 c}$

S-15

16. Figure S7. ${ }^{1} \mathrm{H}$ NMR spectrum of $\mathbf{6 d}$

S-16

17. Figure S8. ${ }^{13} \mathrm{C}$ NMR spectrum of $\mathbf{6 d}$

18. Figure S9. ${ }^{1} \mathrm{H}$ NMR spectrum of $\mathbf{6} \mathbf{f}$

S-18

19. Figure S10. ${ }^{13} \mathrm{C}$ NMR spectrum of $\mathbf{6 f}$

20. Figure S11. ${ }^{1} \mathrm{H}$ NMR spectrum of $\mathbf{6 g}$

21. Figure S12. ${ }^{13} \mathrm{C}$ NMR spectrum of $\mathbf{6 g}$

22. Figure S13. ${ }^{1} \mathrm{H}$ NMR spectrum of $\mathbf{6 h}$

23. Figure S14. ${ }^{13} \mathrm{C}$ NMR spectrum of $\mathbf{6 h}$

24. Figure S15. ${ }^{1} \mathrm{H}$ NMR spectrum of $7 \mathbf{a}$

25. Figure S16. ${ }^{13} \mathrm{C}$ NMR spectrum of $7 \mathbf{a}$

26. Figure S17. ${ }^{1} \mathrm{H}$ NMR spectrum of $8 \mathbf{a}$

27. Figure S18. ${ }^{13} \mathrm{C}$ NMR spectrum of $8 \mathbf{a}$

28. Figure S19. ${ }^{1} \mathrm{H}$ NMR spectrum of $\mathbf{7 b}$

29. Figure S20. ${ }^{13} \mathrm{C}$ NMR spectrum of $7 \mathbf{b}$

30. Figure S21. ${ }^{1} \mathrm{H}$ NMR spectrum of $\mathbf{8 b}$ 
33. Figure S24. ${ }^{13} \mathrm{C}$ NMR spectrum of $\mathbf{7 d}$

34. Figure $\mathrm{S} 25 .{ }^{1} \mathrm{H}$ NMR spectrum of $\mathbf{8 d}$

35. Figure S26. ${ }^{13} \mathrm{C}$ NMR spectrum of $\mathbf{8 d}$

S-36

36. Figure S27. ${ }^{1} \mathrm{H}$ NMR spectrum of $\mathbf{7 e + 8 e}$

S-37

37. Figure S28. ${ }^{13} \mathrm{C}$ NMR spectrum of $\mathbf{7 e}+\mathbf{8 e}$

S-38

38. Figure $S 29 .{ }^{1} \mathrm{H}$ NMR spectrum of $\mathbf{7 f}$

39. Figure $\mathrm{S} 30 .{ }^{13} \mathrm{C}$ NMR spectrum of $\mathbf{7 f}$

40. Figure $S 31 .{ }^{1} \mathrm{H}$ NMR spectrum of $\mathbf{7 g}$

S-41

41. Figure $S 32 .{ }^{13} \mathrm{C}$ NMR spectrum of $\mathbf{7 g}$

$\mathrm{S}-42$

42. Figure $S 33 .{ }^{1} \mathrm{H}$ NMR spectrum of $\mathbf{7 h}$

$\mathrm{S}-43$

43. Figure S34. ${ }^{13} \mathrm{C}$ NMR spectrum of $7 \mathbf{h}$

S-44

44. Figure S35. ${ }^{1} \mathrm{H}$ NMR spectrum of $\mathbf{1 3}$

$\mathrm{S}-45$

45. Figure S36. ${ }^{13} \mathrm{C}$ NMR spectrum of $\mathbf{1 3}$

S-46

46. Figure S37. ${ }^{1} \mathrm{H}$ NMR spectrum of $\mathbf{1 4}$

S-47

47. Figure S38. ${ }^{13} \mathrm{C}$ NMR spectrum of $\mathbf{1 4}$

S-48

48. Figure S39. ${ }^{1} \mathrm{H}$ NMR spectrum of $\mathbf{1 5 a}$

49. Figure S40. ${ }^{13} \mathrm{C}$ NMR spectrum of $\mathbf{1 5 a}$

S-50

50. Figure S41. ${ }^{1} \mathrm{H}$ NMR spectrum of $\mathbf{1 5} \mathbf{b}$

S-51

51. Figure S42. ${ }^{13} \mathrm{C}$ NMR spectrum of $\mathbf{1 5 b}$

S-52 
TABLE S1. ${ }^{1} \mathrm{H}$ NMR $\left(\delta, \mathrm{CDCl}_{3}, 300 \mathrm{MHz}\right)^{\mathrm{a}}$ data of $7 \mathrm{a}$

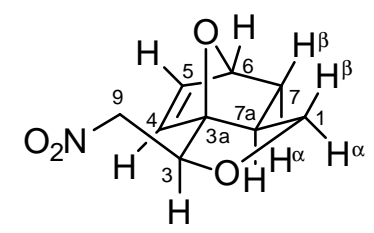

$7 a$

\begin{tabular}{|c|c|c|c|c|c|c|c|c|c|c|}
\hline No. & $1^{\beta}$ & $1^{\mathrm{a}}$ & 3 & 4 & 5 & 6 & $7 \mathrm{a}$ & $7^{\mathrm{a}}$ & $7^{\beta}$ & $\mathrm{CH}_{2} \mathrm{NO}_{2}$ \\
\hline $1^{13}$ & $\begin{array}{l}\mathbf{3 . 5 3} \\
\text { (dd) }\end{array}$ & 8.1 & - & - & - & - & 10.3 & - & - & - \\
\hline $1^{\mathrm{a}}$ & & $\begin{array}{l}\mathbf{4 . 2 0} \\
(\mathrm{t})\end{array}$ & - & - & - & - & 8.1 & - & - & - \\
\hline 3 & & & $\begin{array}{l}\mathbf{5 . 0 7} \\
\text { (ddd) }\end{array}$ & - & - & 1.1 & - & - & - & $\begin{array}{l}7.7 \\
4.8\end{array}$ \\
\hline 4 & & & & $\begin{array}{l}\mathbf{6 . 4 6} \\
(\mathrm{m})\end{array}$ & b & - & - & - & - & - \\
\hline 5 & & & & & $\begin{array}{l}\mathbf{6 . 4 6} \\
(\mathrm{m})\end{array}$ & b & - & - & - & - \\
\hline 6 & & & & & & $\begin{array}{l}\mathbf{5 . 1 3} \\
\text { (dd) }\end{array}$ & - & - & 4.0 & - \\
\hline $7 \mathrm{a}$ & & & & & & & $\begin{array}{l}\mathbf{2 . 3 0} \\
\text { (dddd) }\end{array}$ & 7.3 & 2.9 & - \\
\hline $7^{\mathrm{a}}$ & & & & & & & & $\begin{array}{l}\mathbf{1 . 4 3} \\
\text { (dd) }\end{array}$ & 11.7 & - \\
\hline $7^{\mathrm{B}}$ & & & & & & & & & $\begin{array}{l}\mathbf{1 . 7 9} \\
\text { (ddd) }\end{array}$ & - \\
\hline $\mathrm{CH}_{2} \mathrm{NO}_{2}$ & & & & & & & & & & $\begin{array}{l}\mathbf{4 . 8 1} \\
(\mathrm{ABX})\end{array}$ \\
\hline
\end{tabular}

${ }^{\text {a }}$ Chemical shifts and multiplicities (in parentheses) are placed diagonally and $J(\mathrm{~Hz})$ offdiagonally (upper); confirmed by COSY (400 MHz) analysis; ${ }^{b}$ Overlapped. 
TABLE S2. ${ }^{1} \mathrm{H}$ NMR $\left(\delta, \mathbf{C}_{6} \mathrm{D}_{6}, 300 \mathrm{MHz}\right)^{\mathrm{a}}$ data of $8 \mathrm{a}$

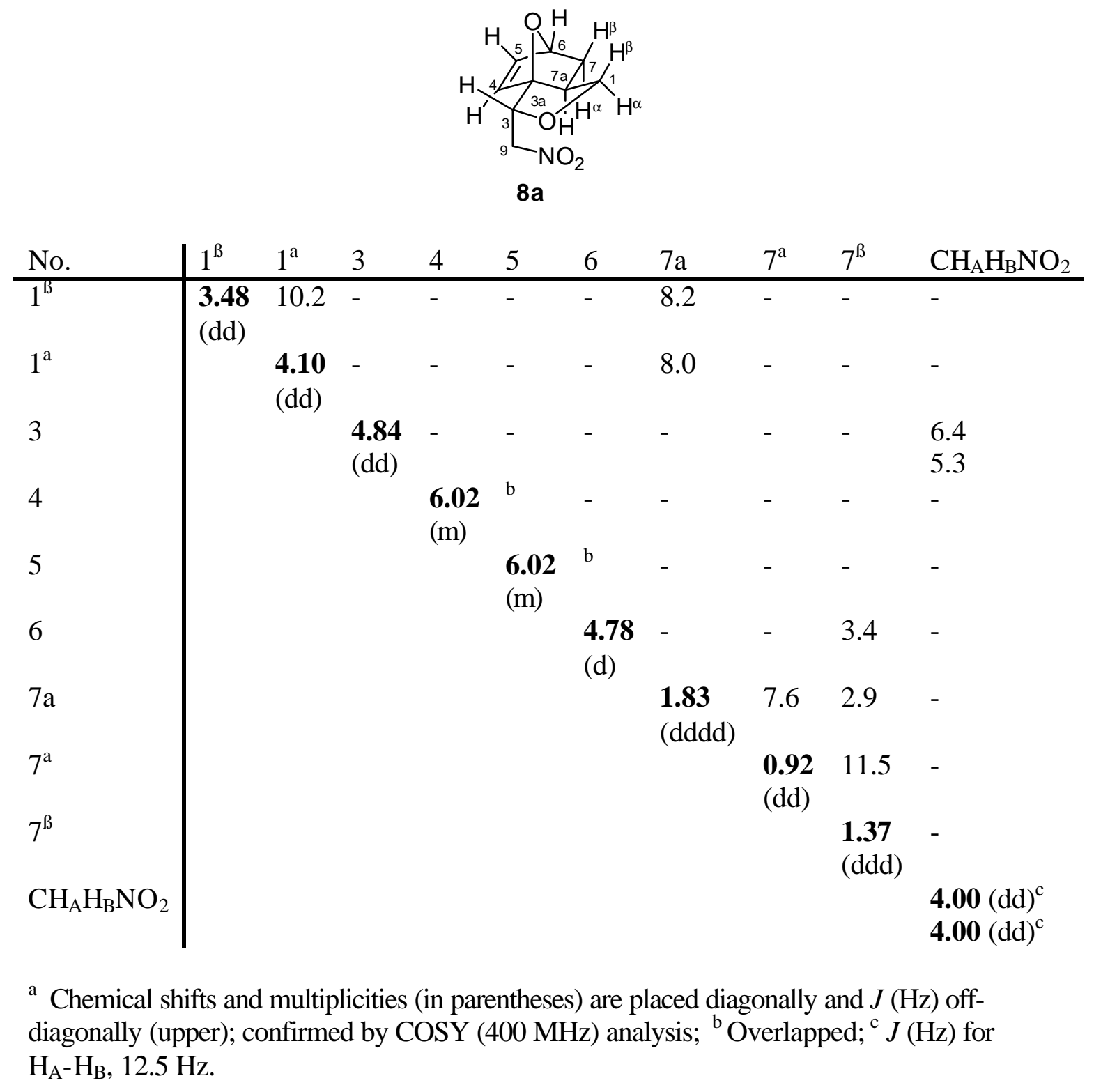


TABLE S3. ${ }^{1} \mathrm{H}$ NMR $\left(\delta, \mathrm{CDCl}_{3}, 300 \mathrm{MHz}\right)^{\mathrm{a}}$ and ROESY $(400 \mathrm{MHz})^{\mathrm{b}}$ data of $7 \mathrm{~b}$

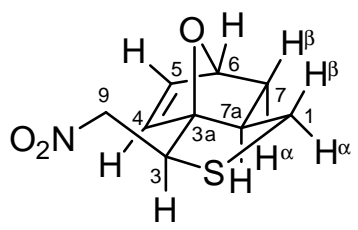

$7 b$

\begin{tabular}{|c|c|c|c|c|c|c|c|c|c|c|}
\hline No. & $1^{\beta}$ & $1^{\mathrm{a}}$ & 3 & 4 & 5 & 6 & $7 a$ & $7^{\mathrm{a}}$ & $7^{\beta}$ & $\mathrm{CH}_{2} \mathrm{NO}_{2}$ \\
\hline $1^{13}$ & $\begin{array}{l}2.77 \\
(\mathrm{t})\end{array}$ & 10.7 & - & - & - & - & 10.7 & - & - & - \\
\hline $1^{\mathrm{a}}$ & $\mathrm{S}$ & $\begin{array}{l}\mathbf{3 . 0 1} \\
\text { (dd) }\end{array}$ & - & - & - & - & 7.3 & - & - & - \\
\hline 3 & - & - & $\begin{array}{l}\mathbf{4 . 7 5} \\
\text { (dd) }\end{array}$ & - & - & - & - & - & - & $\begin{array}{l}17.1 \\
9.6\end{array}$ \\
\hline 4 & - & - & W & $\begin{array}{l}\mathbf{6 . 3 6} \\
(\mathrm{ABq})\end{array}$ & 5.7 & - & - & - & - & - \\
\hline 5 & - & - & - & - & $\begin{array}{l}6.36 \\
(A B q)\end{array}$ & 1.2 & - & - & - & - \\
\hline 6 & - & - & - & - & - & $\begin{array}{l}\mathbf{5 . 0 3} \\
\text { (dd) }\end{array}$ & - & - & 4.4 & - \\
\hline $7 \mathrm{a}$ & M & $S$ & - & - & - & - & $\begin{array}{l}\mathbf{2 . 3 8} \\
\text { (dddd) }\end{array}$ & 7.4 & 2.5 & - \\
\hline $7^{\mathrm{a}}$ & - & - & - & - & - & - & M & $\begin{array}{l}\mathbf{1 . 4 7} \\
\text { (dd) }\end{array}$ & 11.7 & - \\
\hline $7^{\beta}$ & - & - & - & - & - & W & - & $S$ & $\begin{array}{l}\mathbf{1 . 7 6} \\
\text { (ddd) }\end{array}$ & - \\
\hline $\mathrm{CH}_{2} \mathrm{NO}_{2}$ & - & - & $S$ & - & - & - & - & - & - & $\begin{array}{l}\mathbf{4 . 5 0} \\
(\mathrm{m})\end{array}$ \\
\hline
\end{tabular}

${ }^{\text {a }}$ Chemical shifts and multiplicities (in parentheses) are placed diagonally and $J(\mathrm{~Hz})$ offdiagonally (upper); confirmed by COSY (400 MHz) analysis; ${ }^{b}$ Placed off-diagonally (lower), S: Strong; M: Medium: W: Weak 
TABLE S4. ${ }^{1} \mathrm{H}$ NMR $\left(\delta, \mathrm{CDCl}_{3}, 300 \mathrm{MHz}\right)^{\mathrm{a}}$ and $\mathrm{ROESY}^{\mathrm{b}}$ data of $8 \mathrm{~b}$

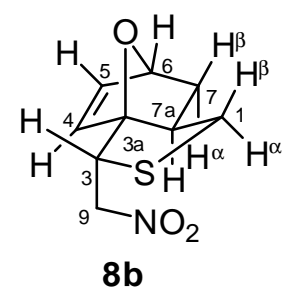

\begin{tabular}{|c|c|c|c|c|c|c|c|c|c|c|}
\hline No. & $1^{\beta}$ & $1^{\mathrm{a}}$ & 3 & 4 & 5 & 6 & $7 \mathrm{a}$ & $7^{\mathrm{a}}$ & $7^{\beta}$ & $\mathrm{CH}_{\mathrm{A}} \mathrm{H}_{\mathrm{B}} \mathrm{NO}_{2}$ \\
\hline $1^{B}$ & $\begin{array}{l}\mathbf{3 . 1 4} \\
\text { (dd) }\end{array}$ & 10.5 & - & - & - & - & 11.0 & - & - & - \\
\hline $1^{\mathrm{a}}$ & $\mathrm{S}$ & $\begin{array}{l}\mathbf{2 . 7 8} \\
(\mathrm{t})\end{array}$ & - & - & - & - & 10.5 & - & - & - \\
\hline 3 & - & - & $\begin{array}{l}\mathbf{4 . 3 0} \\
\text { (dd) }\end{array}$ & - & - & - & - & - & - & $\begin{array}{l}7.5 \\
7.0\end{array}$ \\
\hline 4 & - & - & - & $\begin{array}{l}6.28 \\
\text { (d) }\end{array}$ & 6.0 & - & - & - & - & - \\
\hline 5 & - & - & - & $\mathrm{M}$ & $\begin{array}{l}\mathbf{6 . 5 2} \\
\text { (dd) }\end{array}$ & 1.5 & - & - & - & - \\
\hline 6 & - & - & - & - & - & $\begin{array}{l}\mathbf{5 . 0 7} \\
\text { (dd) }\end{array}$ & - & - & 4.5 & - \\
\hline $7 \mathrm{a}$ & $\mathrm{M}$ & $S$ & - & - & - & - & $\begin{array}{l}\mathbf{2 . 3 5} \\
\text { (dddd) }\end{array}$ & 7.5 & 2.5 & - \\
\hline $7^{\mathrm{a}}$ & - & - & - & - & - & - & $\mathrm{S}$ & $\begin{array}{l}\mathbf{1 . 5 6} \\
\text { (dd) }\end{array}$ & 12.0 & - \\
\hline $7^{\beta}$ & - & W & - & - & - & W & W & $S$ & $\begin{array}{l}\mathbf{1 . 8 3} \\
\text { (ddd) }\end{array}$ & - \\
\hline $\mathrm{CH}_{\mathrm{A}} \mathrm{H}_{\mathrm{B}} \mathrm{NO}_{2}$ & - & - & $\begin{array}{l}S \\
S\end{array}$ & $\begin{array}{l}\text { W } \\
\text { W }\end{array}$ & - & - & - & - & & $\begin{array}{l}4.61(\mathrm{dd})^{\mathrm{c}} \\
\mathbf{4 . 8 5}(\mathrm{dd})^{\mathrm{c}}\end{array}$ \\
\hline
\end{tabular}

${ }^{a}$ Chemical shifts and multiplicities (in parentheses) are placed diagonally and $J(\mathrm{~Hz})$ offdiagonally (upper); confirmed by COSY (400 MHz) analysis; ${ }^{\mathrm{b}}$ Placed off-diagonally (lower), S: Strong; M: Medium: W: Weak; ${ }^{\mathrm{c}} J(\mathrm{~Hz})$ for $\mathrm{H}_{\mathrm{A}}-\mathrm{H}_{\mathrm{B}}, 14.0 \mathrm{~Hz}$. 
TABLE S5. ${ }^{1} \mathrm{H}$ NMR $\left(\delta, \mathrm{CDCl}_{3}, 300 \mathrm{MHz}\right)^{\mathrm{a}}$ data of $7 \mathrm{~d}$

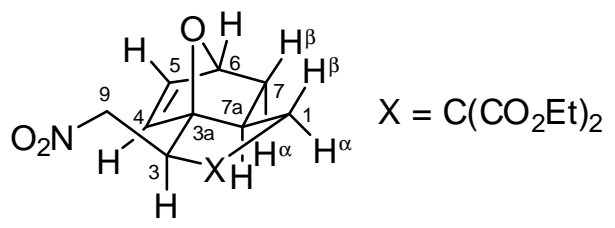

7d

\begin{tabular}{|c|c|c|c|c|c|c|c|c|c|c|c|c|}
\hline No. & $1^{\beta}$ & $1^{\mathrm{a}}$ & 3 & 4 & 5 & 6 & $7 \mathrm{a}$ & $7^{\mathrm{a}}$ & $7^{\beta}$ & $\mathrm{OCH}_{2}$ & $\mathrm{CH}_{3}$ & $\mathrm{CH}_{2} \mathrm{NO}_{2}$ \\
\hline $1^{13}$ & \begin{tabular}{|l|}
$\mathbf{2 . 3 9}$ \\
(dd)
\end{tabular} & 8.5 & - & - & - & - & 8.8 & - & - & - & - & - \\
\hline $1^{\mathrm{a}}$ & & $\begin{array}{l}\mathbf{2 . 3 9} \\
\text { (dd) }\end{array}$ & - & - & - & - & 8.5 & - & - & - & - & - \\
\hline 3 & & & $\begin{array}{l}4.10 \\
(t)\end{array}$ & - & - & - & - & - & - & - & - & 7.1 \\
\hline 4 & & & & $\begin{array}{l}6.29 \\
\text { (d) }\end{array}$ & 5.8 & - & - & - & - & - & - & - \\
\hline 5 & & & & & $\begin{array}{l}6.21 \\
\text { (dd) }\end{array}$ & 1.1 & - & - & - & - & - & - \\
\hline 6 & & & & & & $\begin{array}{l}4.93 \\
\text { (dd) }\end{array}$ & - & - & 3.2 & - & - & - \\
\hline $7 a$ & & & & & & & $\begin{array}{l}\mathbf{1 . 8 7} \\
\text { (dddd) }\end{array}$ & 7.8 & 4.2 & - & - & - \\
\hline $7^{\mathrm{a}}$ & & & & & & & & $\begin{array}{l}\mathbf{1 . 4 8} \\
\text { (dd) }\end{array}$ & 11.4 & - & - & - \\
\hline $7^{\beta}$ & & & & & & & & & $\begin{array}{l}\mathbf{1 . 6 9} \\
\text { (ddd) }\end{array}$ & - & - & - \\
\hline $\mathrm{OCH}_{2}$ & & & & & & & & & & $\begin{array}{l}\mathbf{4 . 1 9} \\
(\mathrm{m})\end{array}$ & 7.2 & - \\
\hline $\mathrm{CH}_{3}$ & & & & & & & & & & & $\begin{array}{l}\mathbf{1 . 2 1} \\
(\mathrm{ABq})\end{array}$ & - \\
\hline $\mathrm{CH}_{2} \mathrm{NO}_{2}$ & & & & & & & & & & & & $\begin{array}{l}\mathbf{4 . 6 3} \\
\text { (d) }\end{array}$ \\
\hline
\end{tabular}

${ }^{\text {a }}$ Chemical shifts and multiplicities (in parentheses) are placed diagonally and $J(\mathrm{~Hz})$ offdiagonally (upper); confirmed by COSY (400 MHz) analysis. 
TABLE S6. ${ }^{1} \mathrm{H}$ NMR $\left(\delta, \mathrm{CDCl}_{3}, 400 \mathrm{MHz}\right)^{\mathrm{a}}$ data of $8 \mathrm{~d}$

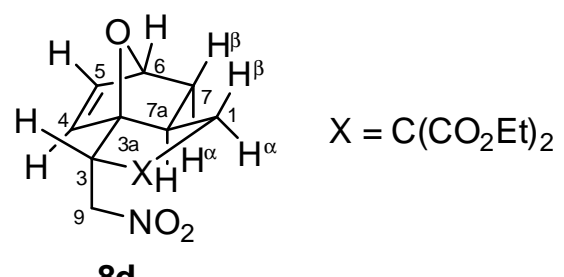

$8 d$

\begin{tabular}{|c|c|c|c|c|c|c|c|c|c|c|c|c|}
\hline No. & $1^{B}$ & $1^{\mathrm{a}}$ & 3 & 4 & 5 & 6 & $7 \mathrm{a}$ & $7^{\mathrm{a}}$ & $7^{\beta}$ & $\mathrm{OCH}_{2}$ & $\mathrm{CH}_{3}$ & $\mathrm{CH}_{\mathrm{A}} \mathrm{H}_{\mathrm{B}} \mathrm{NO}_{2}$ \\
\hline $1^{B}$ & 2.51 & 6.2 & - & - & - & - & 12.8 & - & - & - & - & - \\
\hline $1^{\mathrm{a}}$ & & $\begin{array}{l}\mathbf{2 . 0 7} \\
(\mathrm{m})\end{array}$ & - & - & - & - & b & - & - & - & - & - \\
\hline 3 & & & $\begin{array}{l}\mathbf{3 . 5 0} \\
\text { (dd) }\end{array}$ & - & - & - & - & - & - & - & - & $\begin{array}{l}9.0, \\
4.7\end{array}$ \\
\hline 4 & & & & $\begin{array}{l}\mathbf{6 . 4 0} \\
\text { (d) }\end{array}$ & 5.9 & - & - & - & - & - & - & - \\
\hline 5 & & & & & $\begin{array}{l}\mathbf{6 . 2 9} \\
\text { (dd) }\end{array}$ & 1.6 & - & - & - & - & - & - \\
\hline 6 & & & & & & $\begin{array}{l}\mathbf{5 . 0 0} \\
\text { (dd) }\end{array}$ & - & - & 4.4 & - & - & - \\
\hline $7 \mathrm{a}$ & & & & & & & $\begin{array}{l}\mathbf{2 . 0 7} \\
(\mathrm{m})\end{array}$ & 7.2 & 2.8 & - & - & - \\
\hline $7^{\mathrm{a}}$ & & & & & & & & $\begin{array}{l}1.42 \\
\text { (dd) }\end{array}$ & 11.5 & - & - & - \\
\hline $7^{\beta}$ & & & & & & & & & $\begin{array}{l}\mathbf{1 . 6 9} \\
\text { (ddd) }\end{array}$ & - & - & - \\
\hline $\mathrm{OCH}_{2}$ & & & & & & & & & & $\begin{array}{l}\mathbf{4 . 2 0} \\
(\mathrm{m})\end{array}$ & $\begin{array}{l}7.1 \\
3.4\end{array}$ & - \\
\hline $\mathrm{CH}_{3}$ & & & & & & & & & & & $\begin{array}{l}1.25 \\
\text { (td) }\end{array}$ & - \\
\hline $\mathrm{CH}_{\mathrm{A}} \mathrm{H}_{\mathrm{B}} \mathrm{NO}_{2}$ & & & & & & & & & & & & $\begin{array}{l}\text { 4.90-5.08 } \\
(\mathrm{ABq})^{\mathrm{c}}\end{array}$ \\
\hline
\end{tabular}

${ }^{\text {a }}$ Chemical shifts and multiplicities (in parentheses) are placed diagonally and $J(\mathrm{~Hz})$ offdiagonally (upper); confirmed by COSY $(400 \mathrm{MHz})$ analysis; ${ }^{\mathrm{b}}$ Could not be measured due to overlapping of signals; ${ }^{\mathrm{C}} J(\mathrm{~Hz})$ for $\mathrm{H}_{\mathrm{A}}-\mathrm{H}_{\mathrm{B}}, 14.5 \mathrm{~Hz}$. 
TABLE S7. ${ }^{1} \mathrm{H}$ NMR $\left(\delta, \mathrm{CDCl}_{3}, 300 \mathrm{MHz}\right)^{\mathrm{a}}$ data of $7 \mathrm{e}$

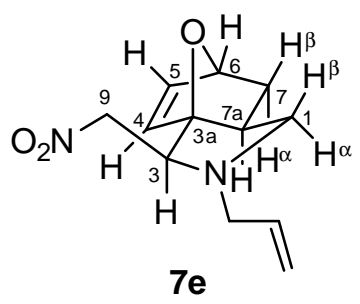

\begin{tabular}{|c|c|c|c|c|c|c|c|c|c|c|c|c|c|}
\hline No. & $1^{\beta}$ & $1^{\mathrm{a}}$ & 3 & 4 & 5 & 6 & $7 \mathrm{a}$ & $7^{\mathrm{a}}$ & $7^{\mathrm{B}}$ & $\underline{\mathrm{CH}_{2}} \mathrm{CH}=$ & $=\mathrm{CH}$ & $=\mathrm{CH}_{\mathrm{A}} \mathrm{H}_{\mathrm{B}}$ & $\mathrm{CH}_{2} \mathrm{NC}$ \\
\hline $1^{15}$ & $\begin{array}{l}2.14 \\
\text { (dd) }\end{array}$ & 8.5 & - & - & - & - & 9.8 & - & - & - & - & - & - \\
\hline $1^{\mathrm{a}}$ & & $\begin{array}{l}\mathbf{3 . 2 9} \\
(\mathrm{dd})\end{array}$ & - & - & - & - & 6.1 & - & - & - & - & - & - \\
\hline 3 & & & $\begin{array}{l}\mathbf{3 . 3 6} \\
(\mathrm{t})\end{array}$ & - & - & - & - & - & - & - & - & - & 4.9 \\
\hline 4 & & & & $\begin{array}{l}\mathbf{6 . 3 8} \\
\text { (d) }\end{array}$ & 6.1 & - & - & - & - & - & - & - & - \\
\hline 5 & & & & & $\begin{array}{l}\mathbf{6 . 2 7} \\
\text { (d) }\end{array}$ & - & - & - & - & - & - & - & - \\
\hline 6 & & & & & & $\begin{array}{l}4.90 \\
\text { (d) }\end{array}$ & - & - & 4.9 & - & - & - & - \\
\hline $7 \mathrm{a}$ & & & & & & & $\begin{array}{l}\text { 1.91- } \\
1.97 \\
\text { (m) }\end{array}$ & 7.3 & b & - & - & - & - \\
\hline $7^{\mathrm{a}}$ & & & & & & & & $\begin{array}{l}\mathbf{1 . 2 7} \\
\text { (dd) }\end{array}$ & 12.2 & - & - & - & - \\
\hline $7^{\beta}$ & & & & & & & & & $\begin{array}{l}\text { 1.57- } \\
1.66 \\
(\mathrm{~m})\end{array}$ & - & - & - & - \\
\hline$\underline{\mathrm{CH}_{2}} \underline{\mathrm{CH}}=$ & & & & & & & & & & $\begin{array}{l}\text { 3.12- } \\
\mathbf{3 . 2 2} \\
(\mathrm{m})\end{array}$ & $\mathrm{b}$ & - & - \\
\hline$=\mathrm{CH}$ & & & & & & & & & & & $\begin{array}{l}\mathbf{5 . 6 9 -} \\
\mathbf{5 . 8 3} \\
(\mathrm{m})\end{array}$ & $\begin{array}{r}17.1 \\
7.3\end{array}$ & - \\
\hline$=\mathrm{CH}_{\mathrm{A}} \mathrm{H}_{\mathrm{B}}$ & & & & & & & & & & & & $\begin{array}{l}\mathbf{5 . 1 3} \\
\text { (dd) }\end{array}$ & - \\
\hline $\mathrm{CH}_{2} \mathrm{NO}_{2}$ & & & & & & & & & & & & & $\begin{array}{l}4.54 \\
(d)^{c}\end{array}$ \\
\hline
\end{tabular}

${ }^{a}$ Chemical shifts and multiplicities (in parentheses) are placed diagonally and $J(\mathrm{~Hz})$ offdiagonally (upper); confirmed by COSY $(400 \mathrm{MHz})$ analysis; ${ }^{\mathrm{b}}$ Could not be measured due to overlapping of signals; ' ${ }^{\mathrm{c}} \mathrm{C}(\mathrm{Hz})$ for $\mathrm{H}_{\mathrm{A}}-\mathrm{H}_{\mathrm{B}}, 7.3 \mathrm{~Hz}$. 
TABLE S8. ${ }^{13} \mathrm{C}$ NMR $\left(\delta, \mathrm{CDCl}_{3}\right)$ Data of 7a-e and 8a-e

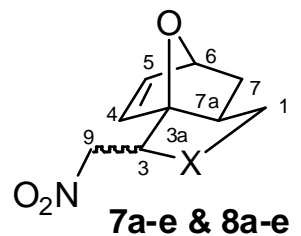

\begin{tabular}{llllllllllll}
\hline No. & $\mathrm{X}$ & $\mathrm{C} 1$ & $\mathrm{C} 3$ & $\mathrm{C} 3 \mathrm{a}$ & $\mathrm{C} 4$ & $\mathrm{C} 5$ & $\mathrm{C} 6$ & $\mathrm{C} 7$ & $\mathrm{C} 7 \mathrm{a}$ & $\mathrm{C} 9$ & Misc. \\
\hline $\mathbf{7 a}$ & $\mathrm{O}$ & 73.2 & 81.0 & 97.5 & 133.1 & 137.5 & 73.3 & 29.7 & 45.4 & 75.0 & - \\
$\mathbf{8 a}$ & $\mathrm{O}$ & 73.5 & 80.4 & 98.8 & 131.7 & 138.1 & 74.5 & 29.3 & 44.5 & 76.9 & - \\
$\mathbf{7 b}$ & $\mathrm{S}$ & 36.8 & 80.3 & 99.0 & 135.3 & 137.6 & 49.7 & 33.8 & 42.5 & 76.1 & - \\
$\mathbf{8 b}$ & $\mathrm{S}$ & 36.6 & 79.4 & 100.6 & 132.1 & 138.9 & 47.3 & 34.5 & 43.8 & 78.0 & - \\
$\mathbf{7 d}$ & $\mathrm{C}\left(\mathrm{CO}_{2} \mathrm{Et}\right)_{2}$ & 38.2 & 79.6 & 96.8 & 134.9 & 136.1 & 42.3 & 33.3 & 40.9 & 72.4 & $13.6,{ }^{\mathrm{a}} 13.8,{ }^{\mathrm{a}} 61.7,{ }^{\mathrm{b}}$ \\
& & & & & & & & & & & $62.0,{ }^{\mathrm{b}} 64.1,{ }^{\mathrm{c}} 168.8,{ }^{\mathrm{d}} 170 . \mathrm{c}$ \\
$\mathbf{8 d}$ & $\mathrm{C}\left(\mathrm{CO}_{2} \mathrm{Et}\right)_{2}$ & 39.1 & 80.2 & 98.2 & 133.5 & 136.4 & 44.5 & 31.6 & 43.2 & 74.2 & $14.0,{ }^{\mathrm{a}} 62.1,{ }^{\mathrm{b}} 66.5,{ }^{\mathrm{c}}$ \\
& & & & & & & & & & & $169.7,{ }^{\mathrm{d}} 170.4^{\mathrm{d}}$ \\
$\mathbf{7 e}$ & $\mathrm{NCH}_{2} \mathrm{CH}=\mathrm{CH}_{2}$ & 41.5 & 79.5 & 96.1 & 135.0 & 136.6 & 58.6 & 30.2 & 57.7 & 76.5 & $61.9,{ }^{\mathrm{e}} 117.6,{ }^{\mathrm{d}} 133.2^{\mathrm{g}}$ \\
$\mathbf{8 e}$ & $\mathrm{NCH}_{2} \mathrm{CH}=\mathrm{CH}_{2}$ & 42.6 & 80.1 & 98.2 & 135.2 & 136.3 & 59.0 & 31.4 & 57.3 & 75.0 & $60.7,{ }^{\mathrm{e}} 117.6,{ }^{\mathrm{f}} 134.7^{\mathrm{g}}$ \\
\hline
\end{tabular}

${ }^{\mathrm{a}}$ methyl, ${ }^{\mathrm{b}} \mathrm{OCH}_{2},{ }^{\mathrm{c}} \mathrm{C}\left(\mathrm{CO}_{2} \mathrm{Et}\right)_{2},{ }^{\mathrm{d}}$ carbonyl, ${ }^{\mathrm{e}} \mathrm{N}-\mathrm{CH}_{2},{ }^{\mathrm{f}}=\mathrm{CH},{ }^{\mathrm{g}}=\mathrm{CH}_{2}$

TABLE S9. ${ }^{13} \mathrm{C}$ NMR $\left(\delta, \mathrm{CDCl}_{3}\right)$ Data of $7 \mathrm{f}, 7 \mathrm{~g}$ and $7 \mathrm{~h}^{\mathrm{a}}$

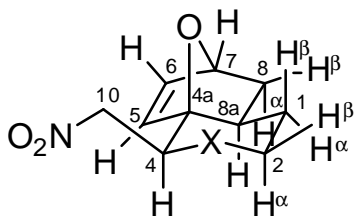

$7 f-h$

\begin{tabular}{llllllllllll}
\hline No. & C1 & C2 & C4 & C4a & C5 & C6 & C7 & C8 & C8a & C10 & Misc. \\
\hline 7f & 34.06 & 34.10 & 79.4 & 84.3 & 133.1 & 138.8 & 67.0 & 31.1 & 29.7 & 74.2 & - \\
7g & 35.0 & 35.9 & 78.9 & 87.6 & 134.6 & 138.6 & 40.7 & 27.4 & 32.6 & 75.4 & $13.9,{ }^{\mathrm{b}} 61.5,{ }^{\mathrm{c}}$ \\
& & & & & & & & & & & $62.3,{ }^{\mathrm{d}} 169.0,{ }^{\mathrm{e}}$ \\
& & & & & & & & & & & $171.2^{\mathrm{e}}$ \\
7h & 31.7 & 35.3 & 78.9 & 86.9 & 135.5 & 138.4 & 38.3 & 24.3 & 26.4 & 78.5 & $35.7^{\mathrm{f}}$ \\
\hline
\end{tabular}

${ }^{a}$ Trivial numbering for $\mathbf{7 f}, \mathbf{7 g}$ and $\mathbf{7 h}$, for systematic numbering: see Experimental Section; ${ }^{\mathrm{b}}$ methyl, ${ }^{\mathrm{c}} \mathrm{OCH}_{2},{ }^{\mathrm{d}} \mathrm{C}\left(\mathrm{CO}_{2} \mathrm{Et}\right)_{2},{ }^{\mathrm{e}}$ carbonyl, ${ }^{\mathrm{f}} \mathrm{CH}_{2}$ 


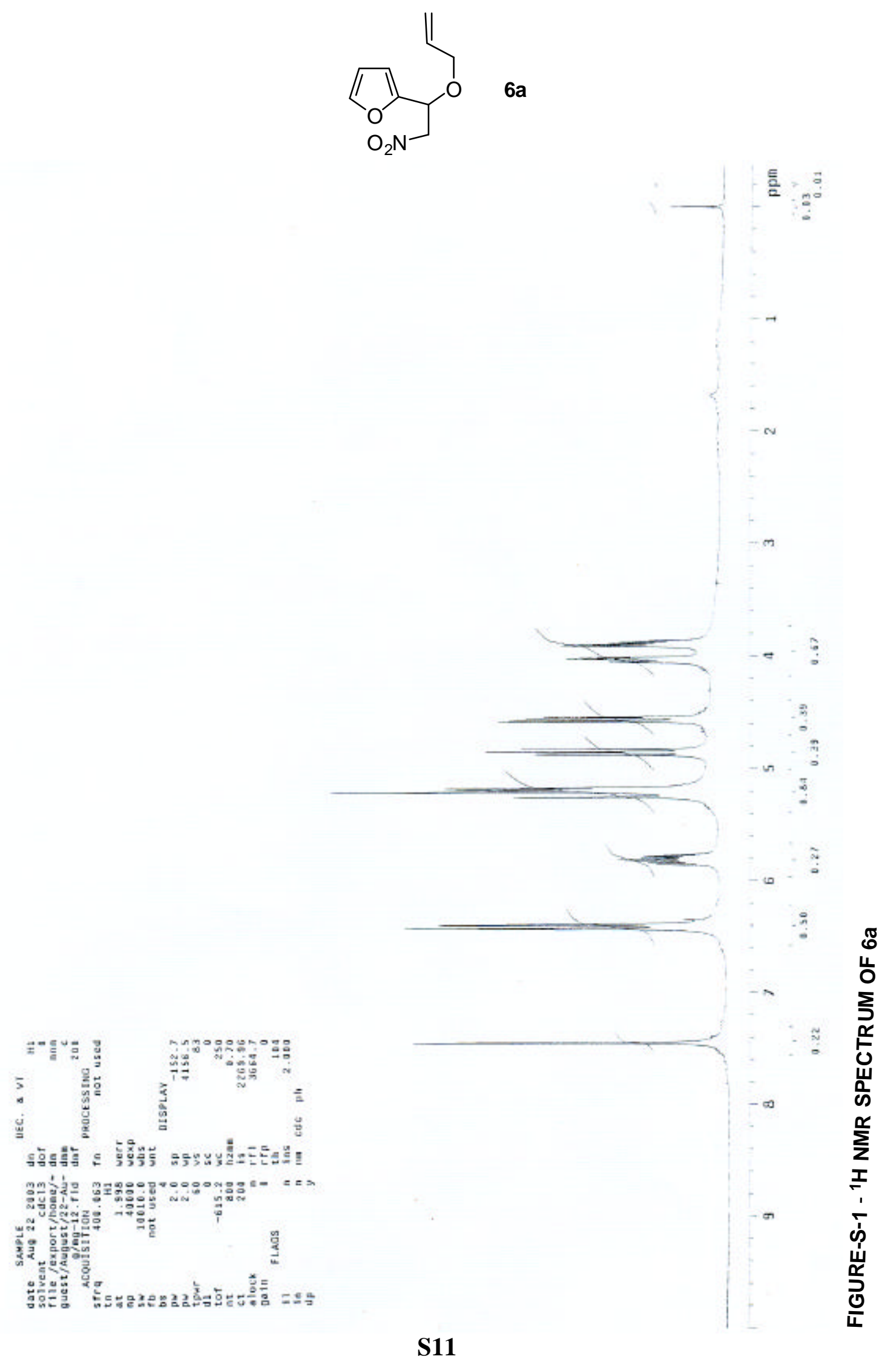




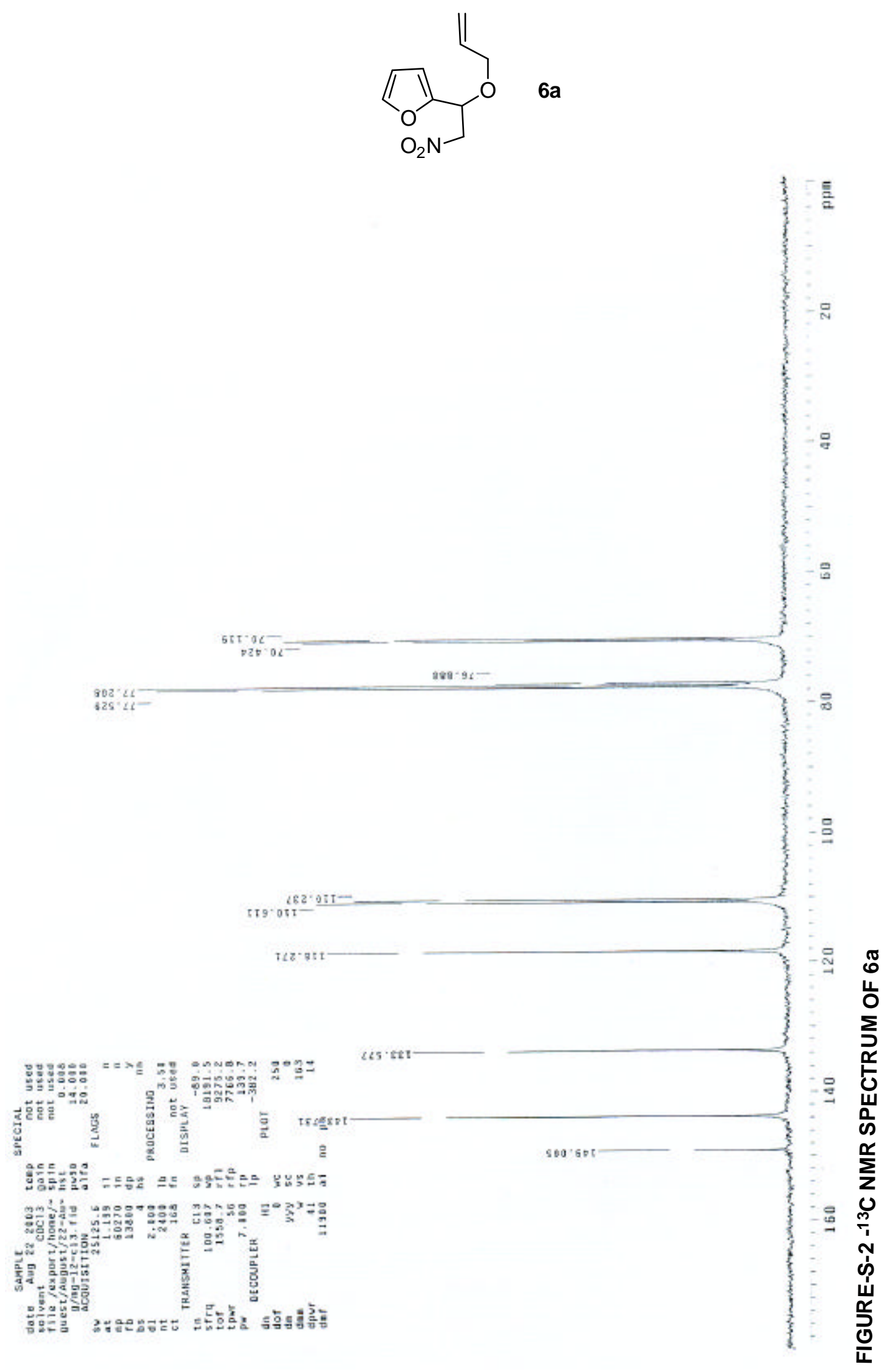

S12 


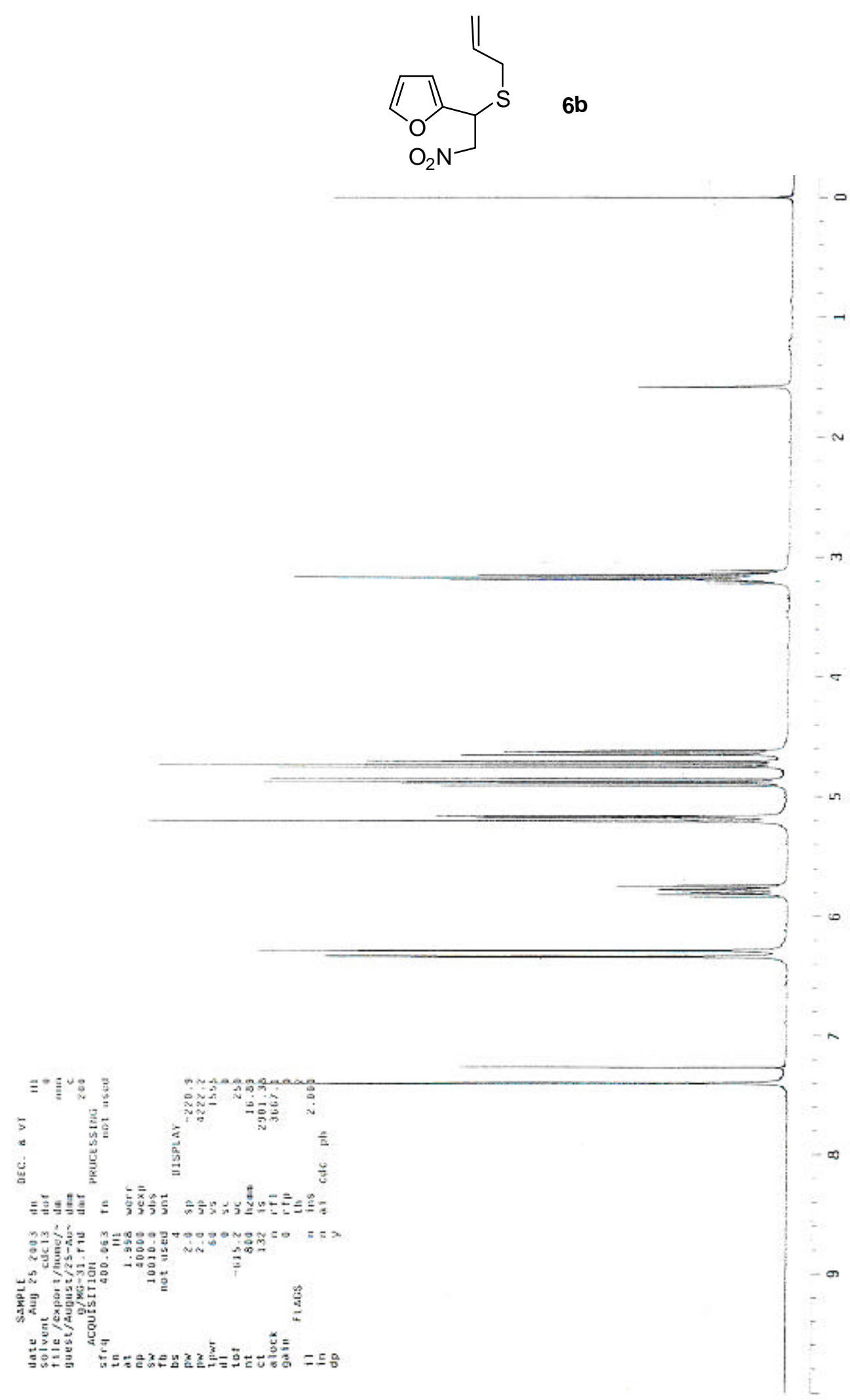

S13 


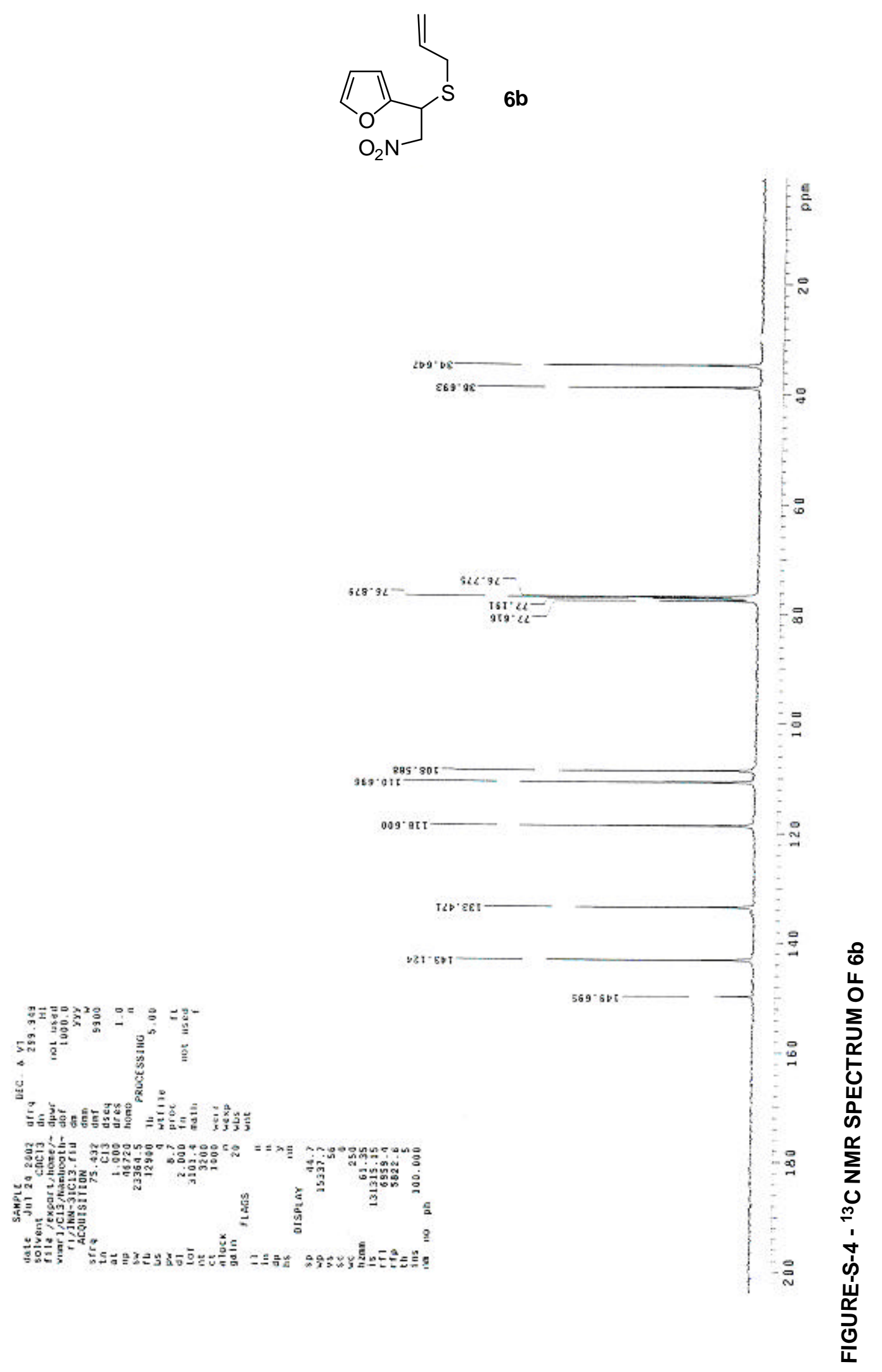

S14 


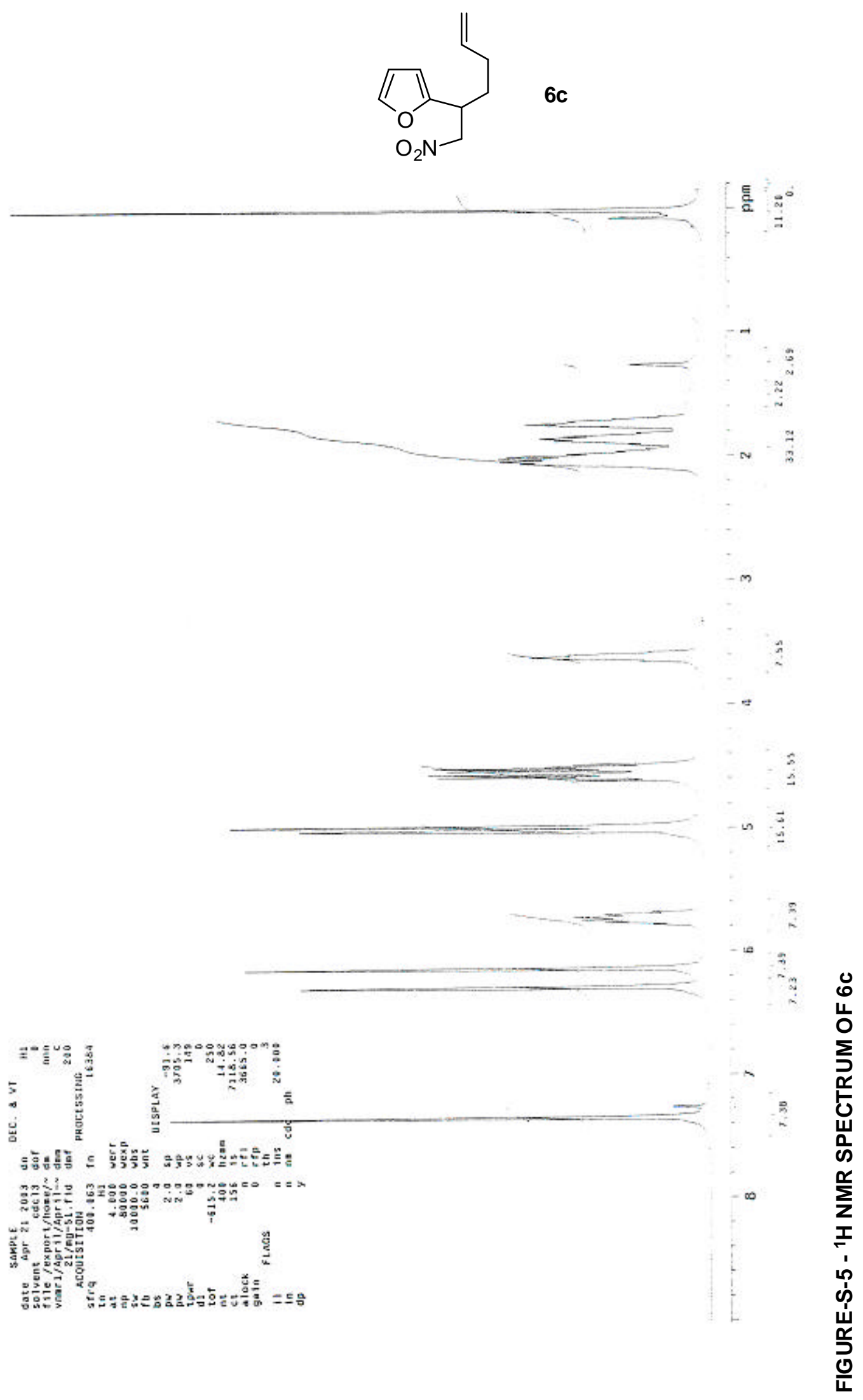

S15 


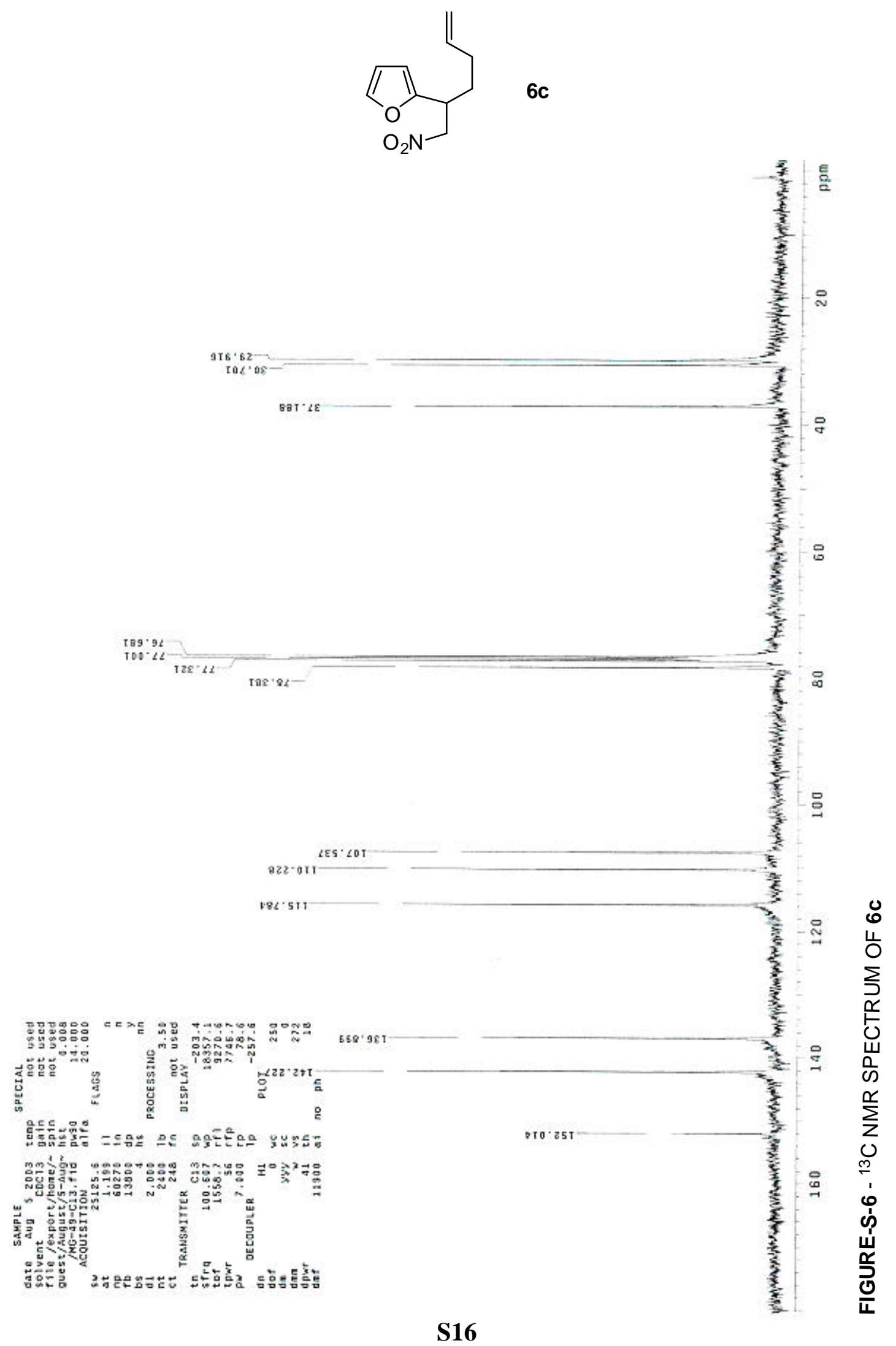




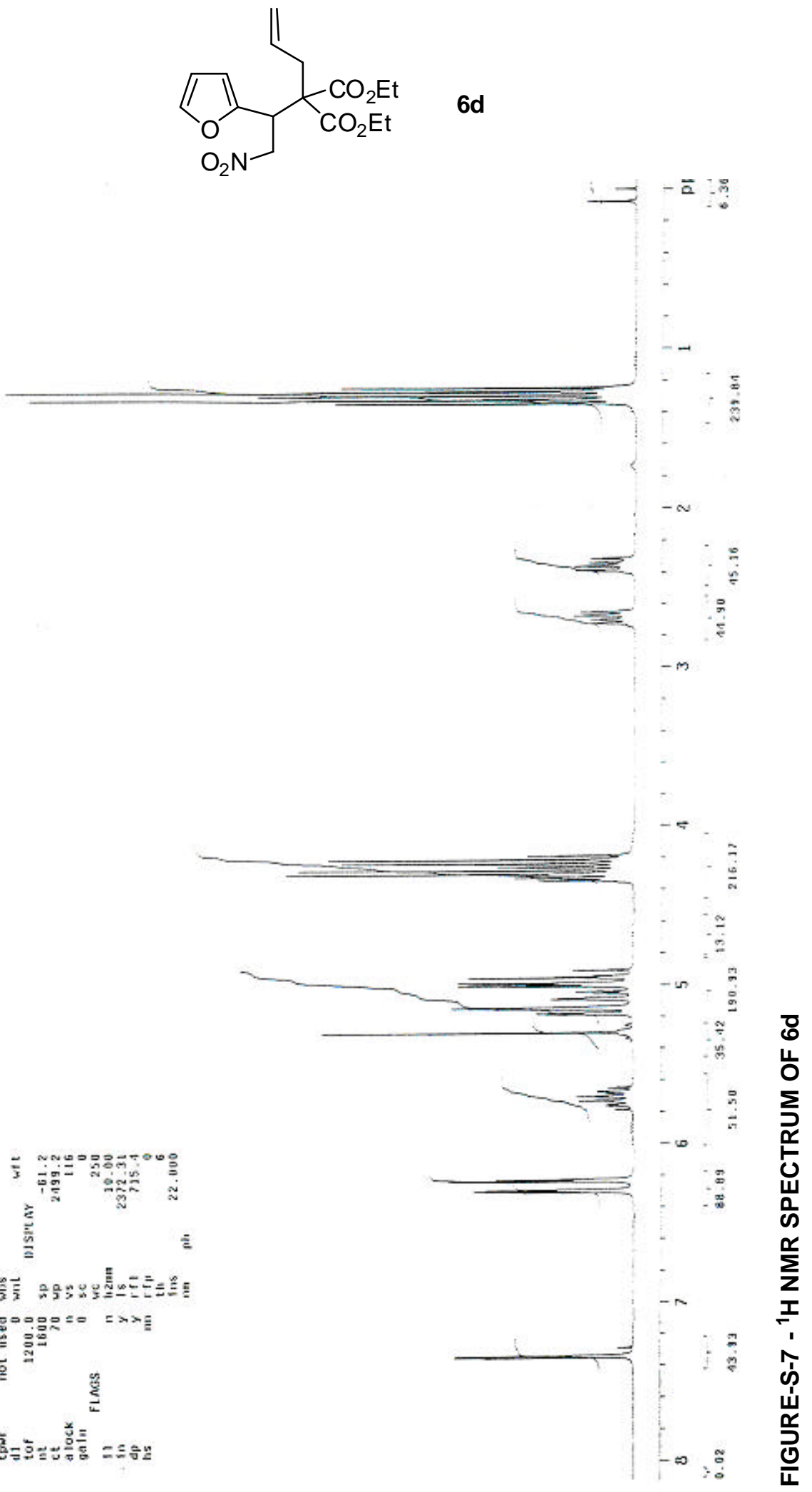




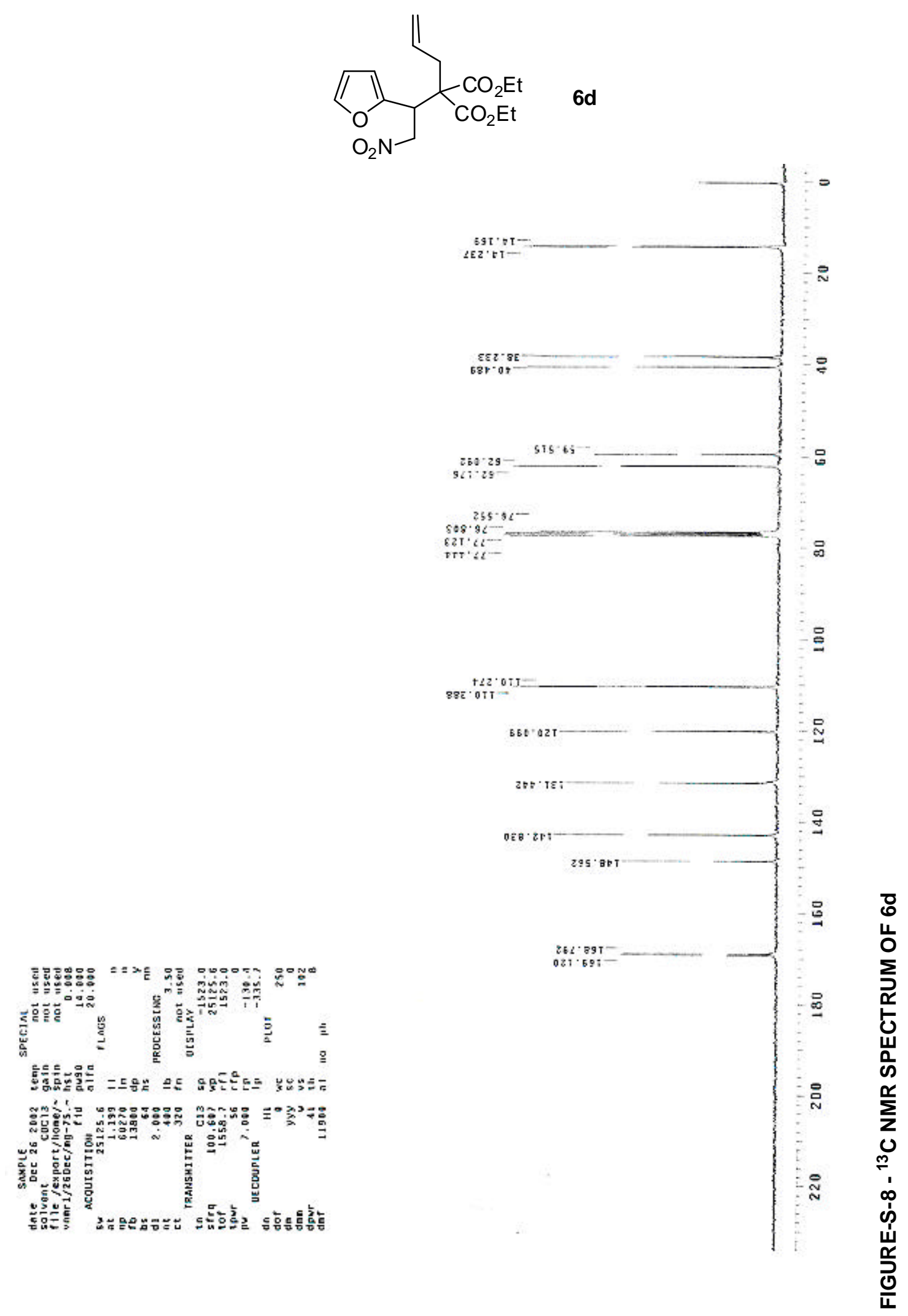

S18 


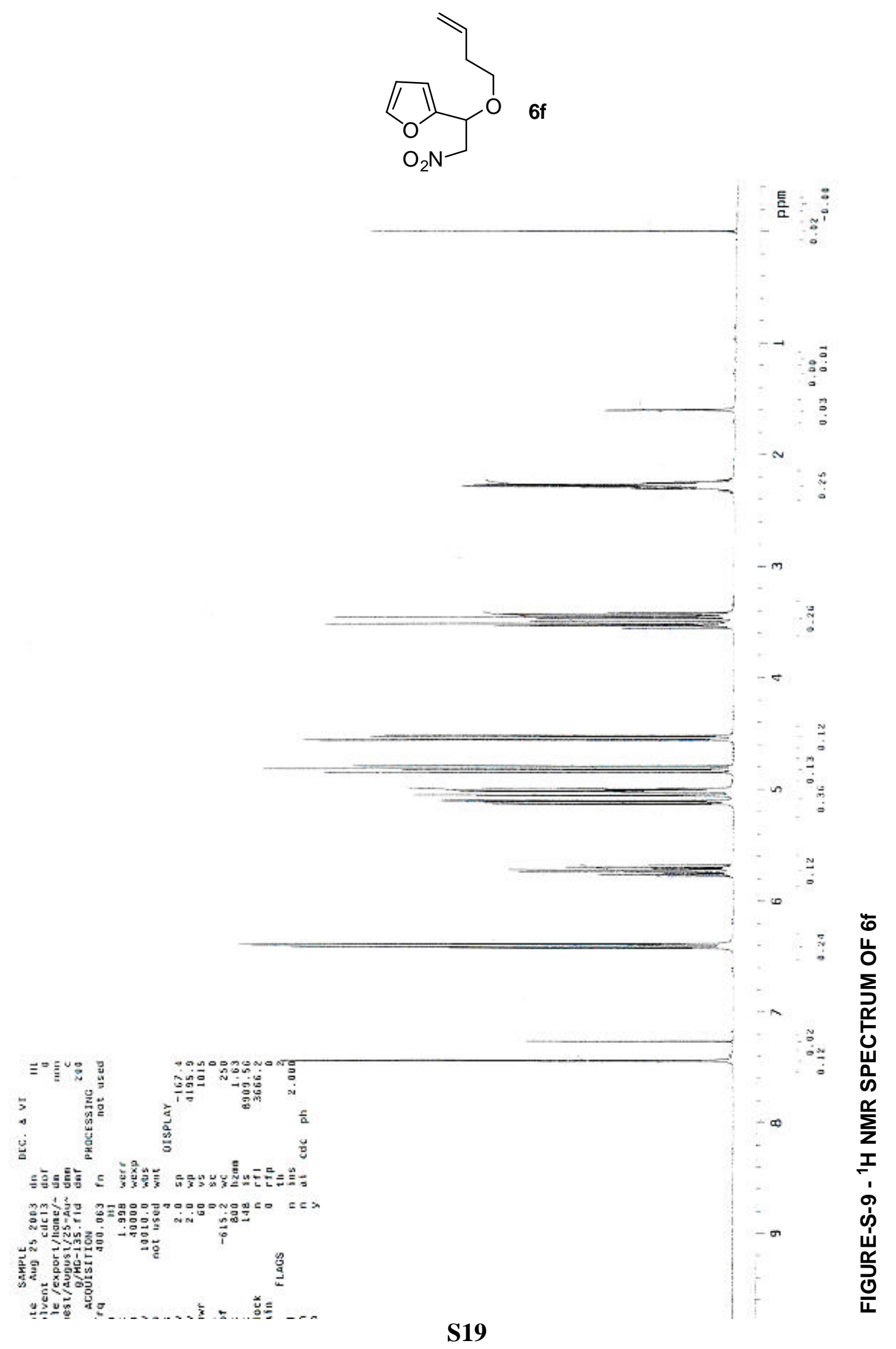




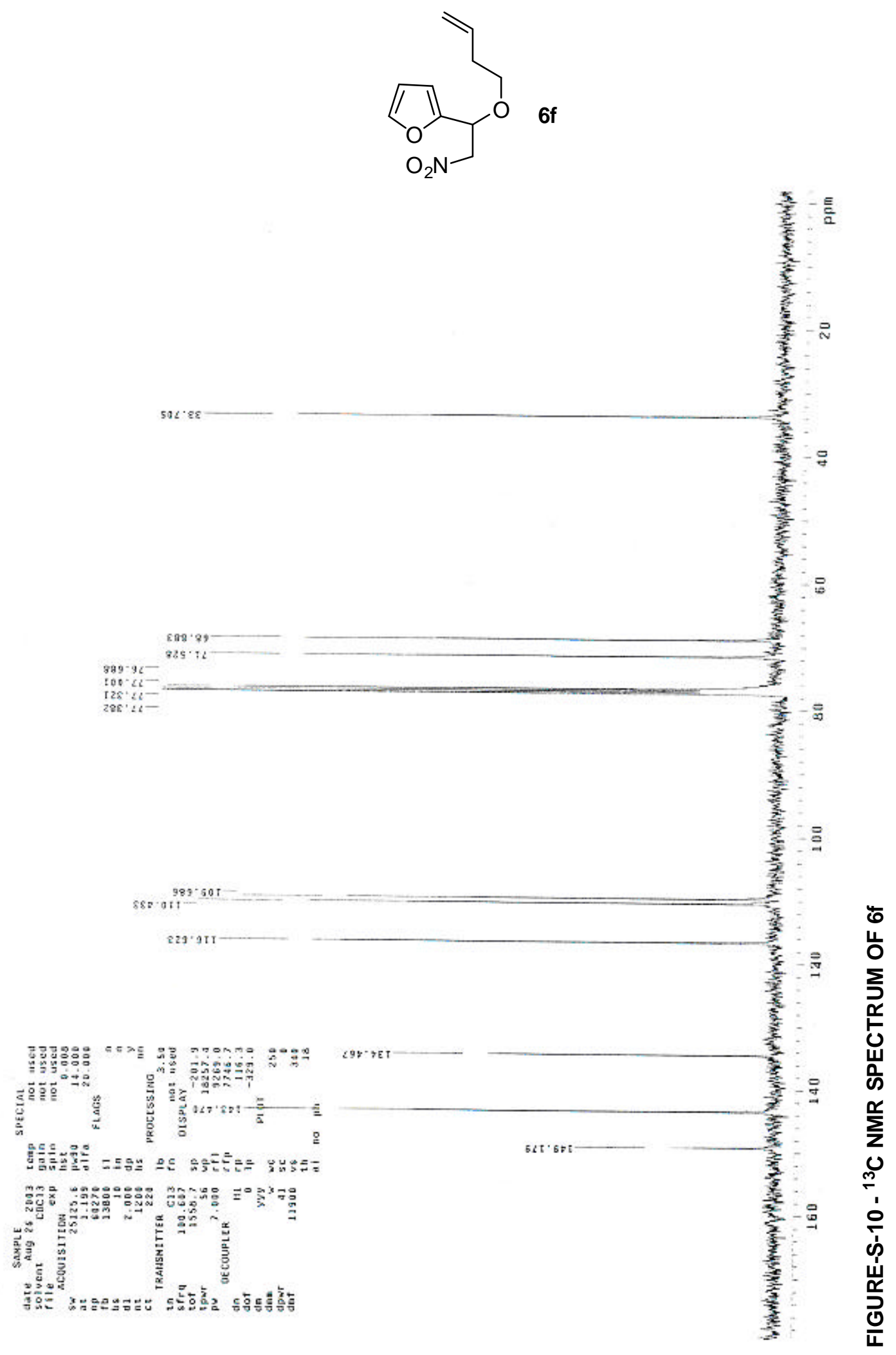



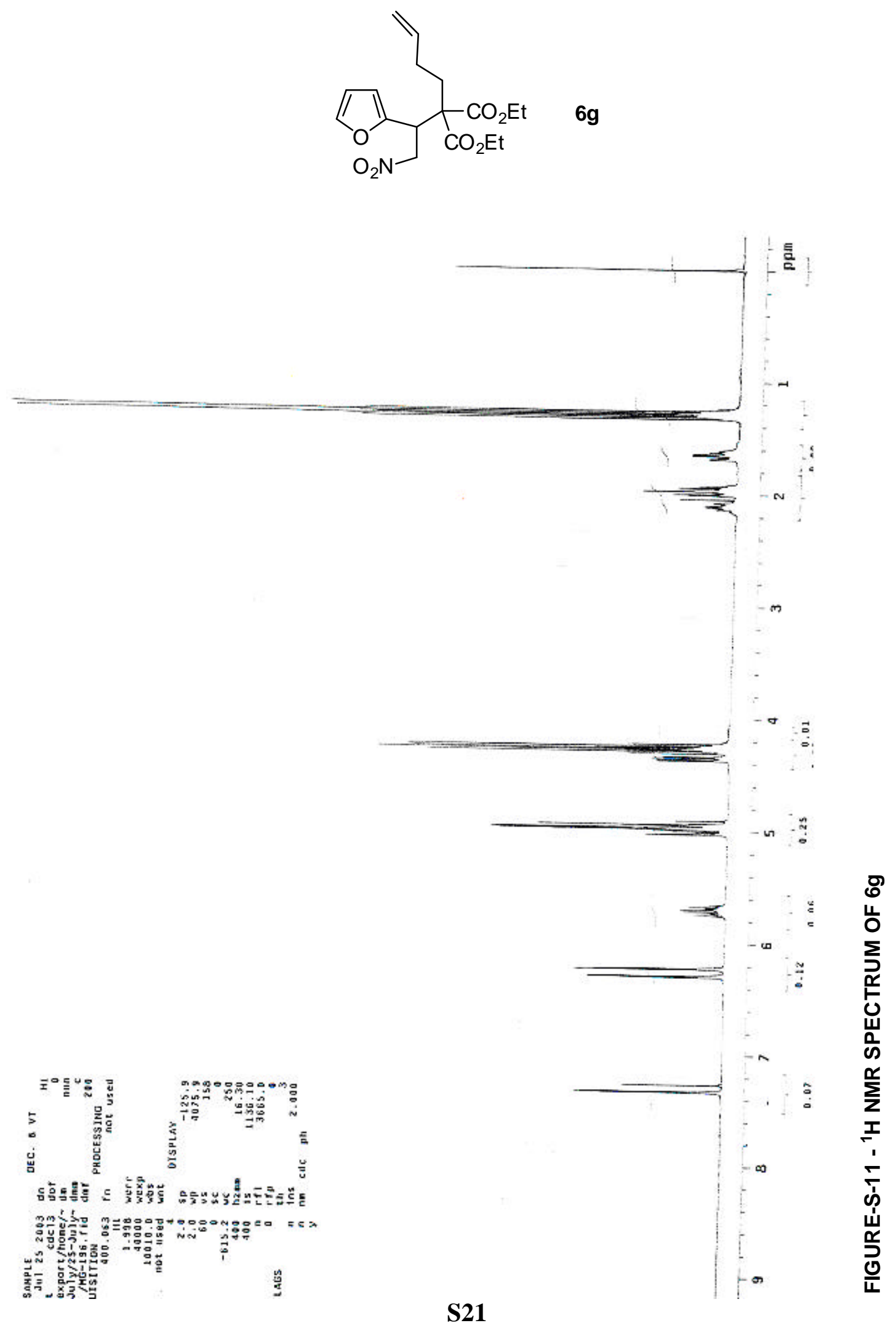


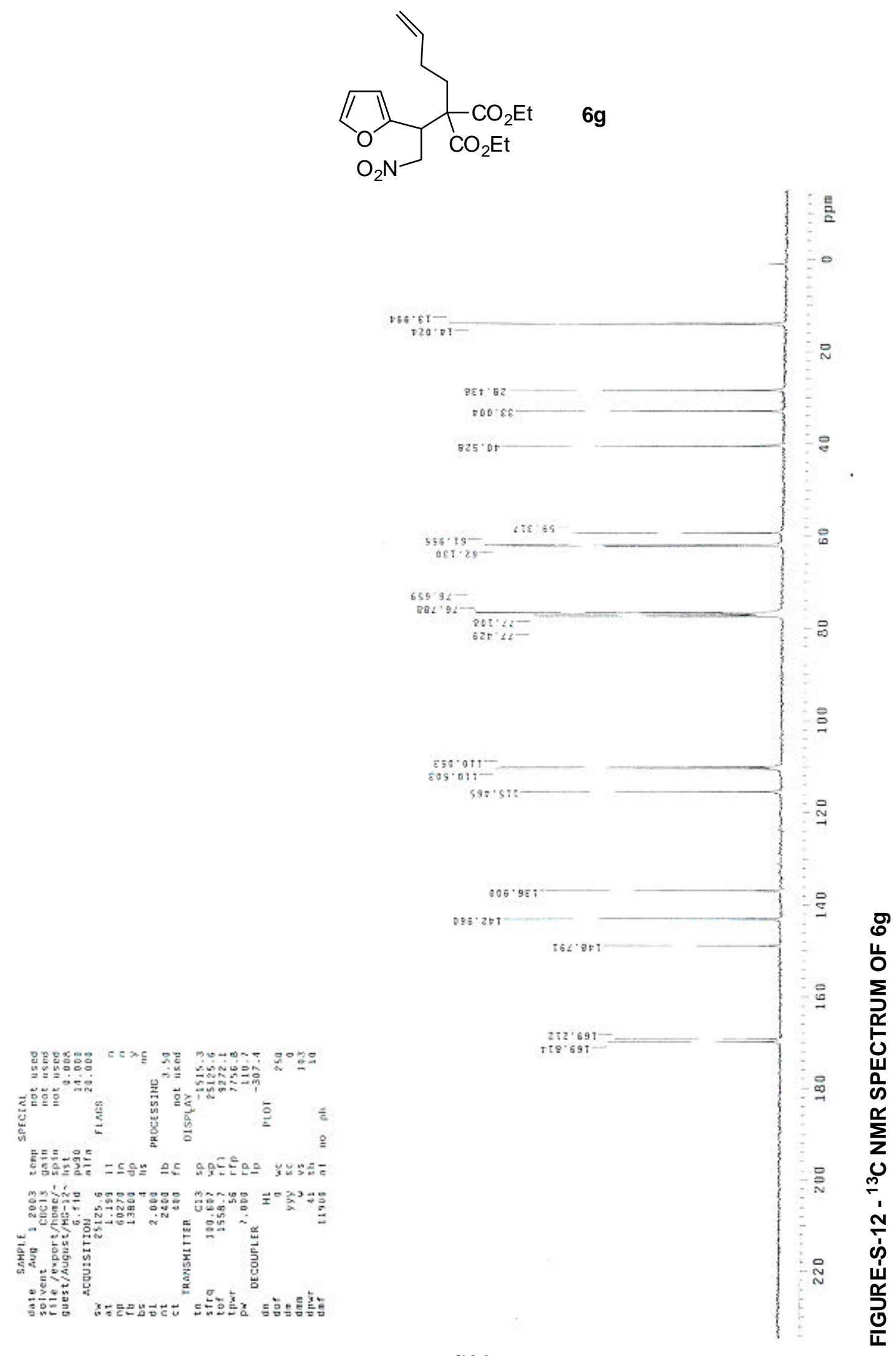




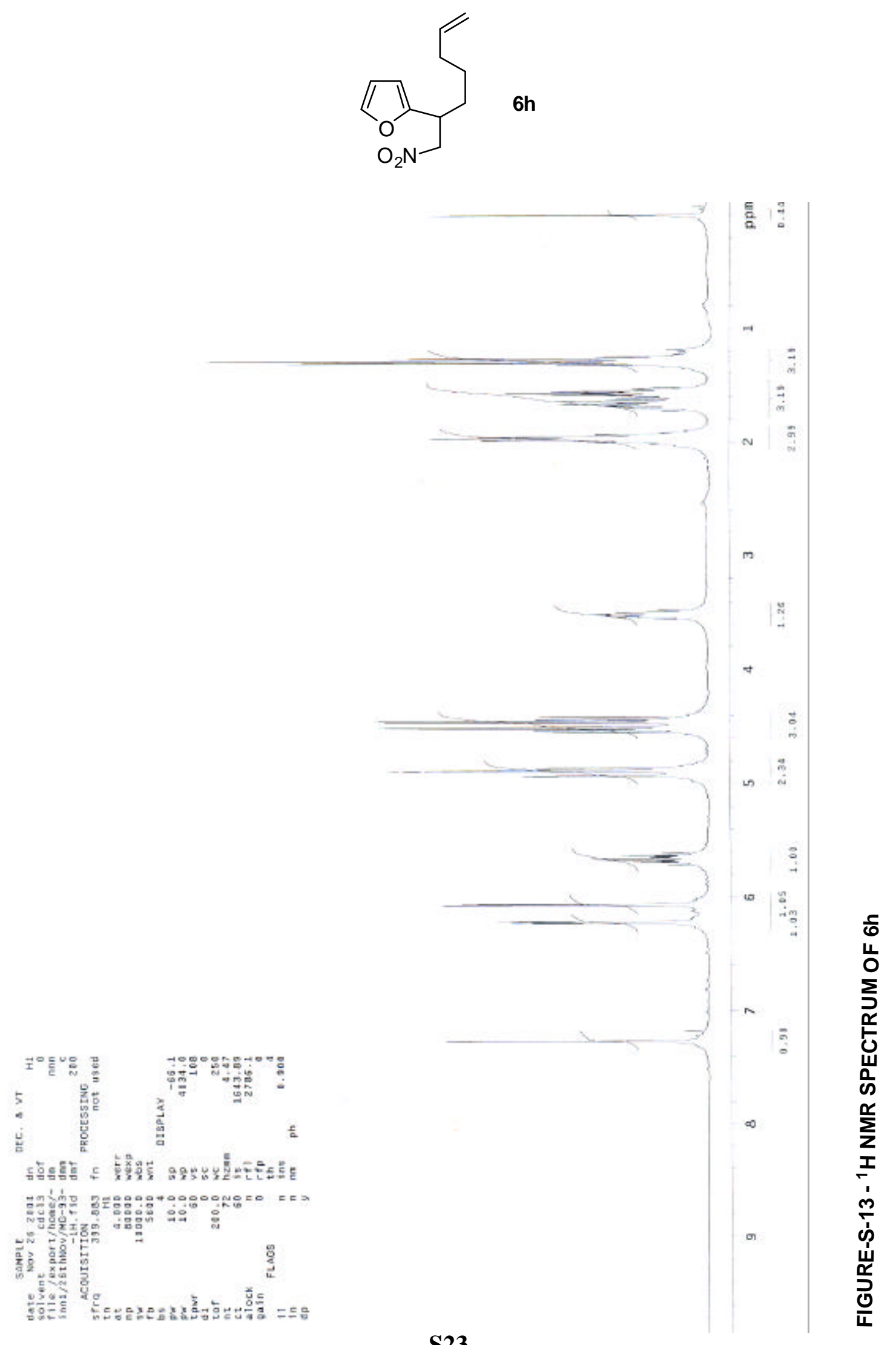




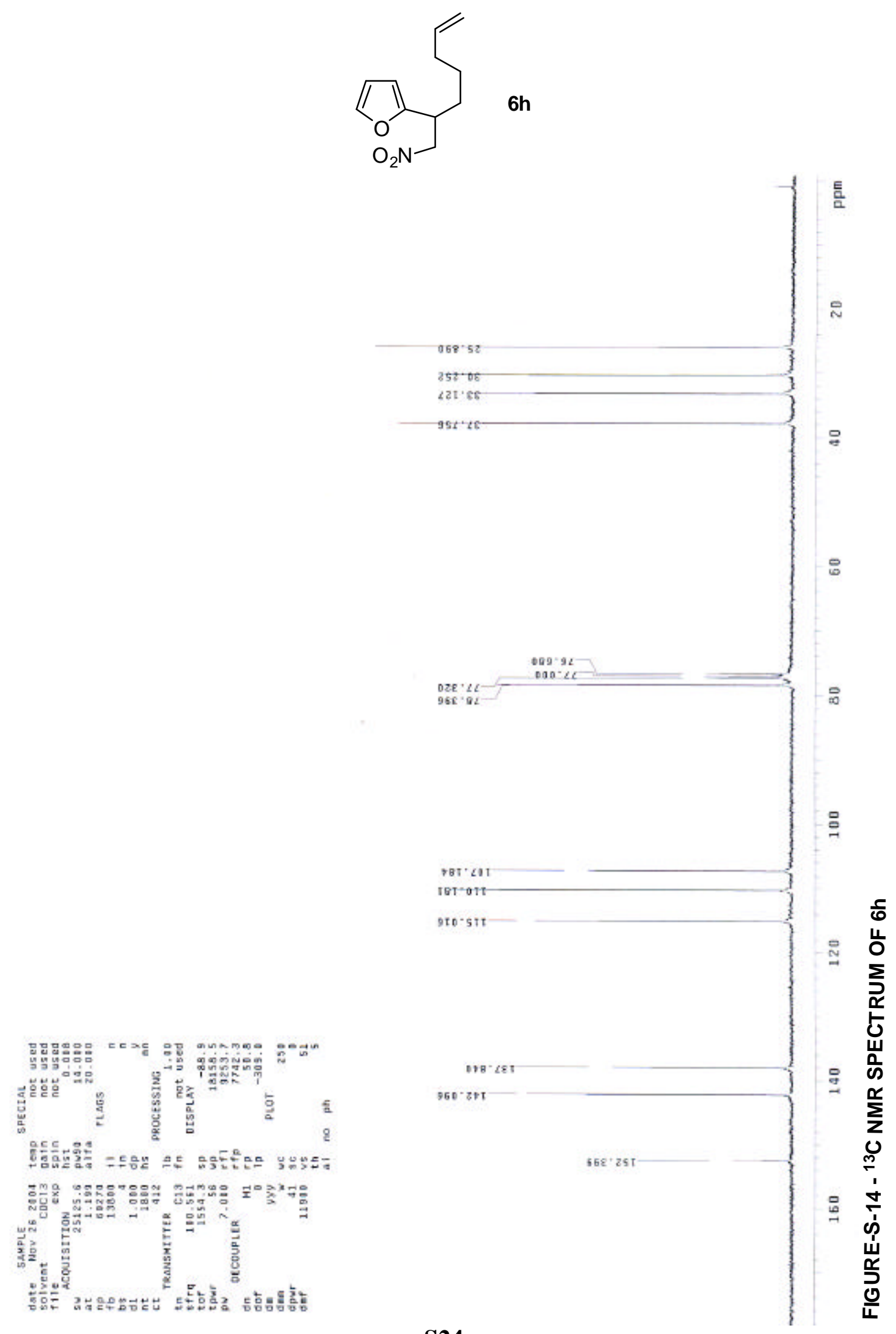




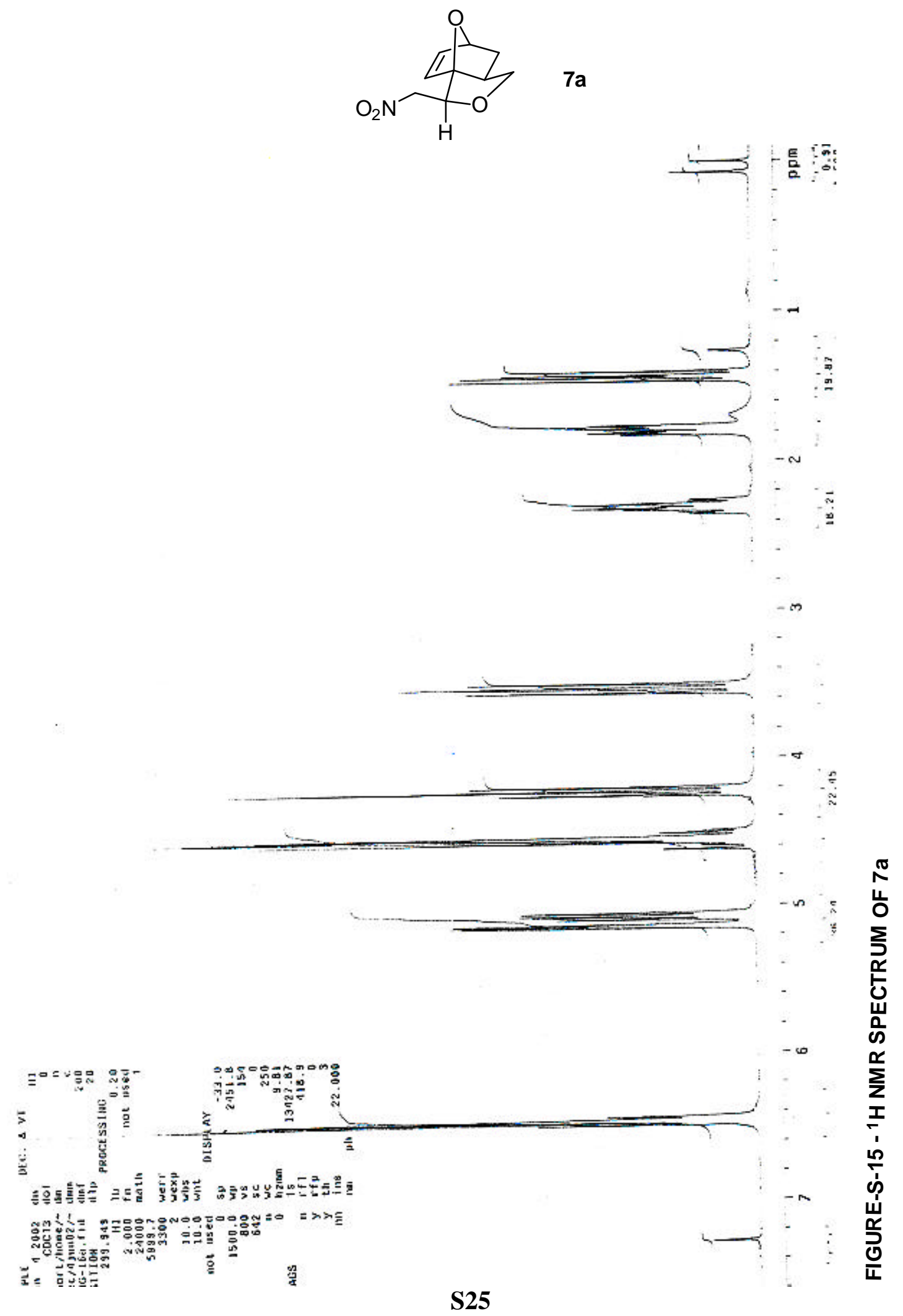




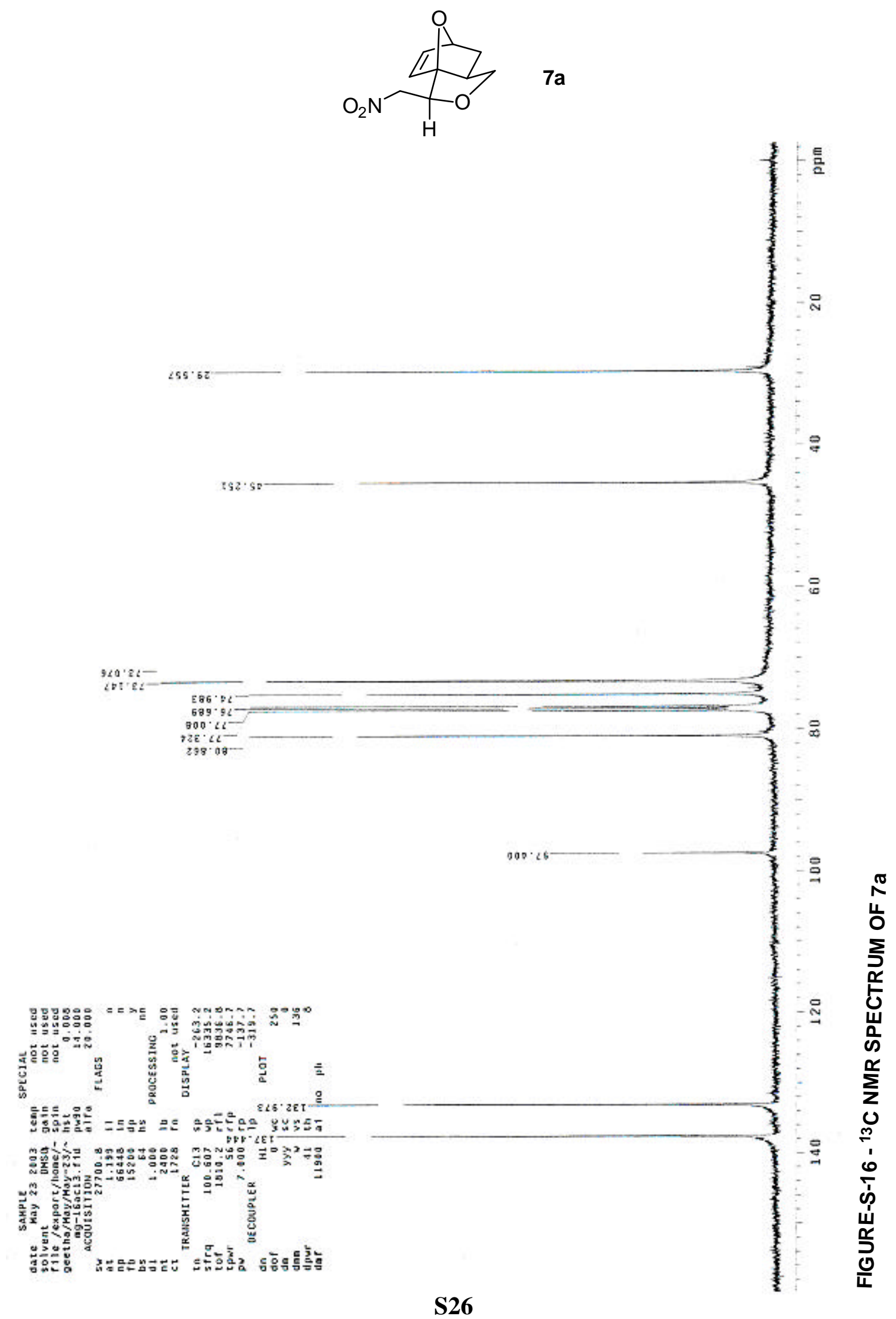



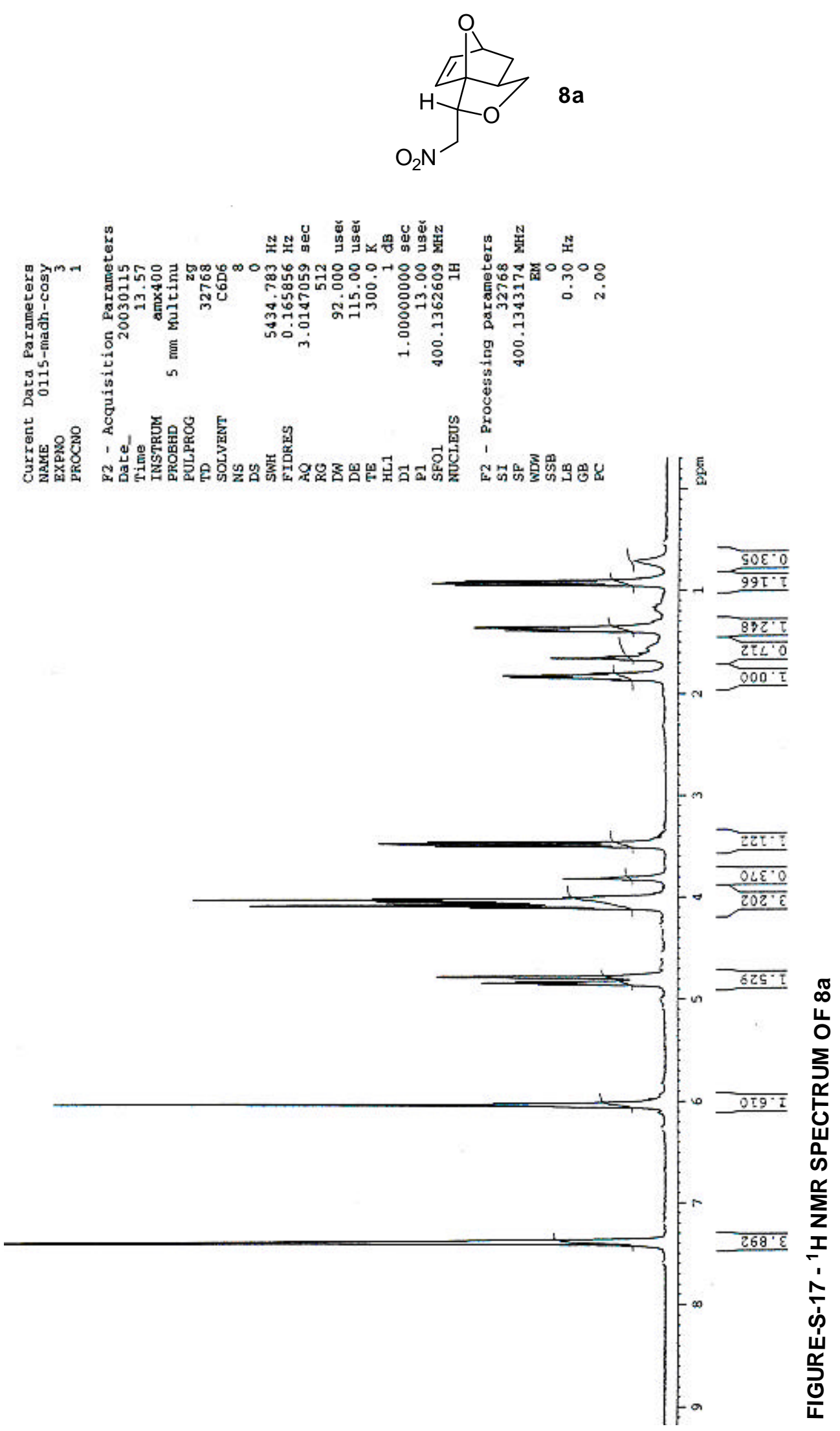

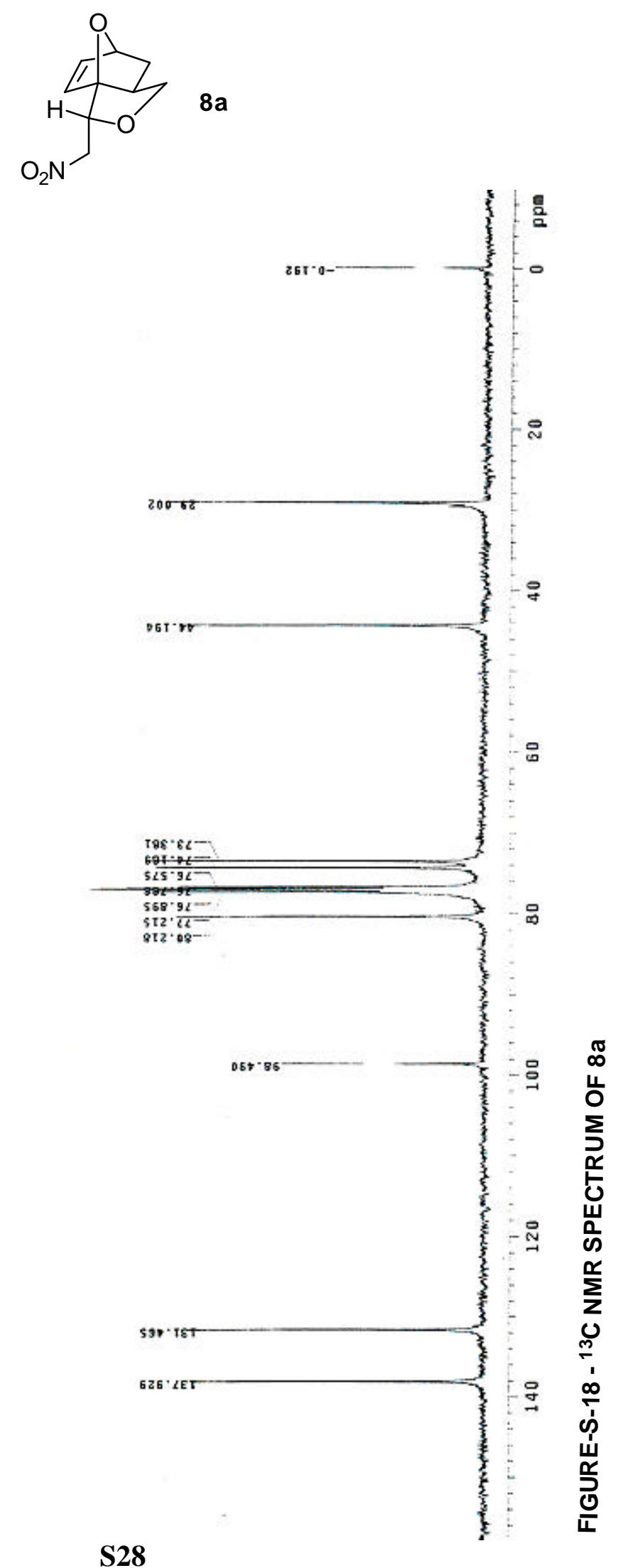


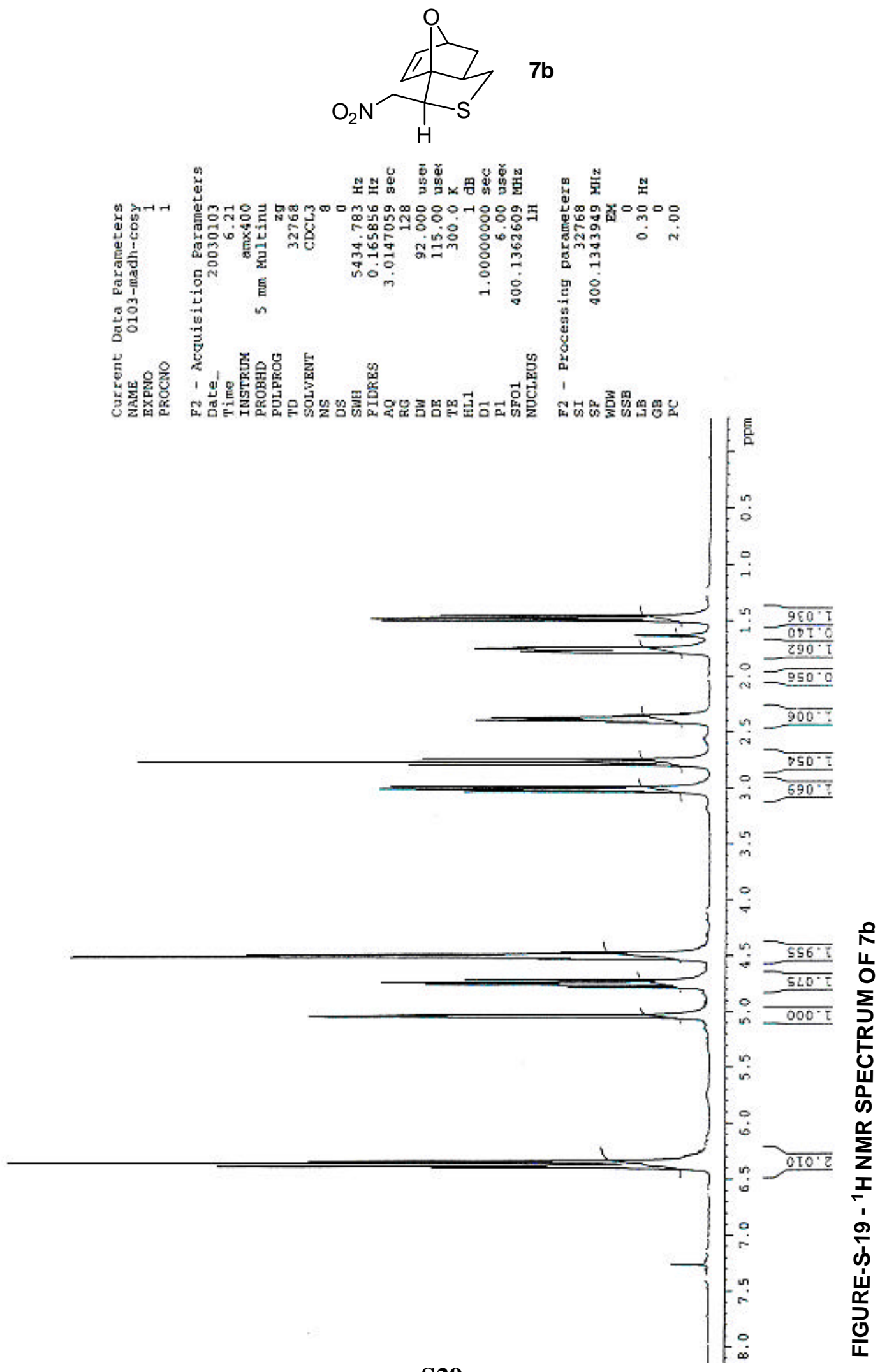




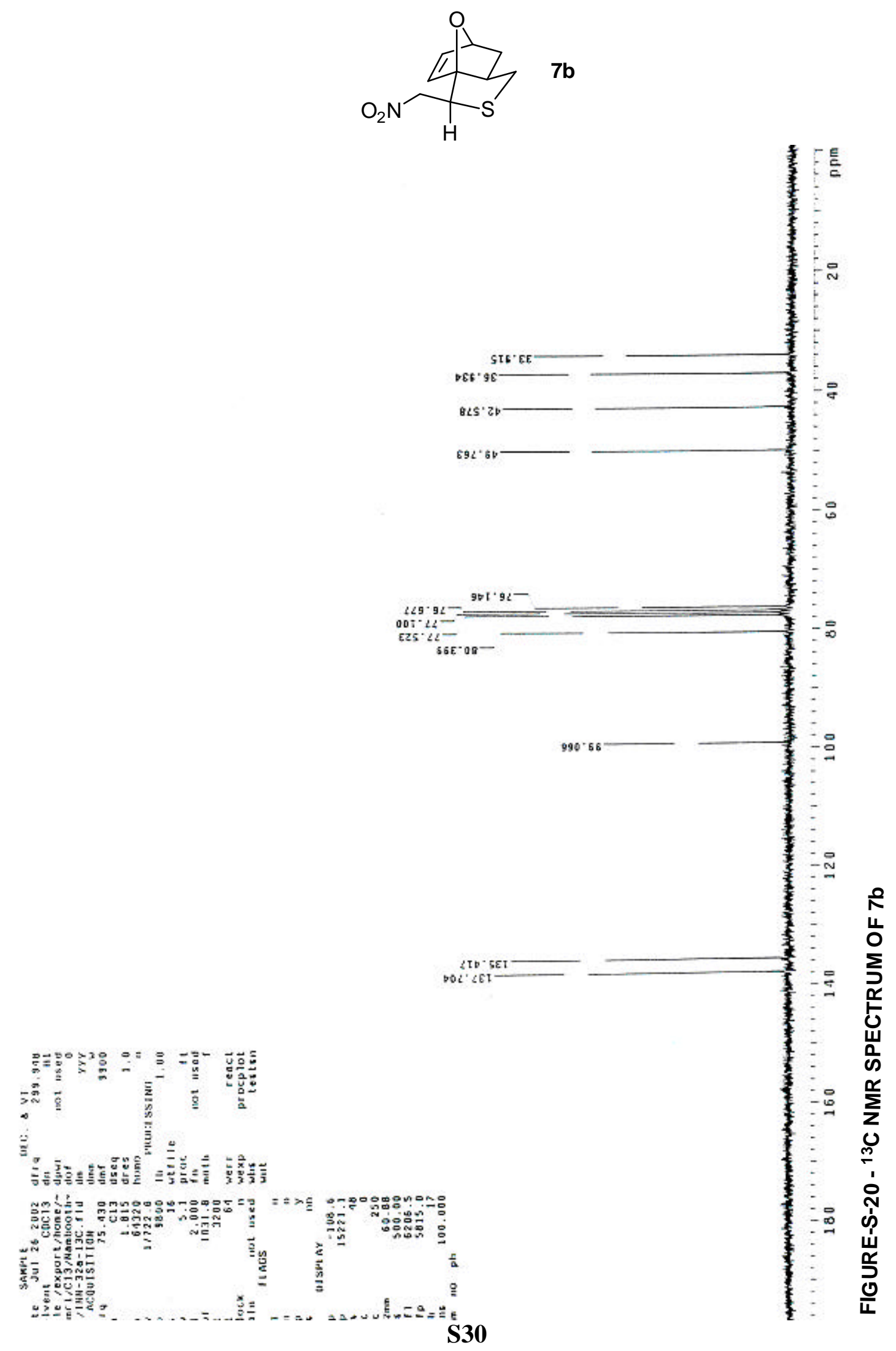



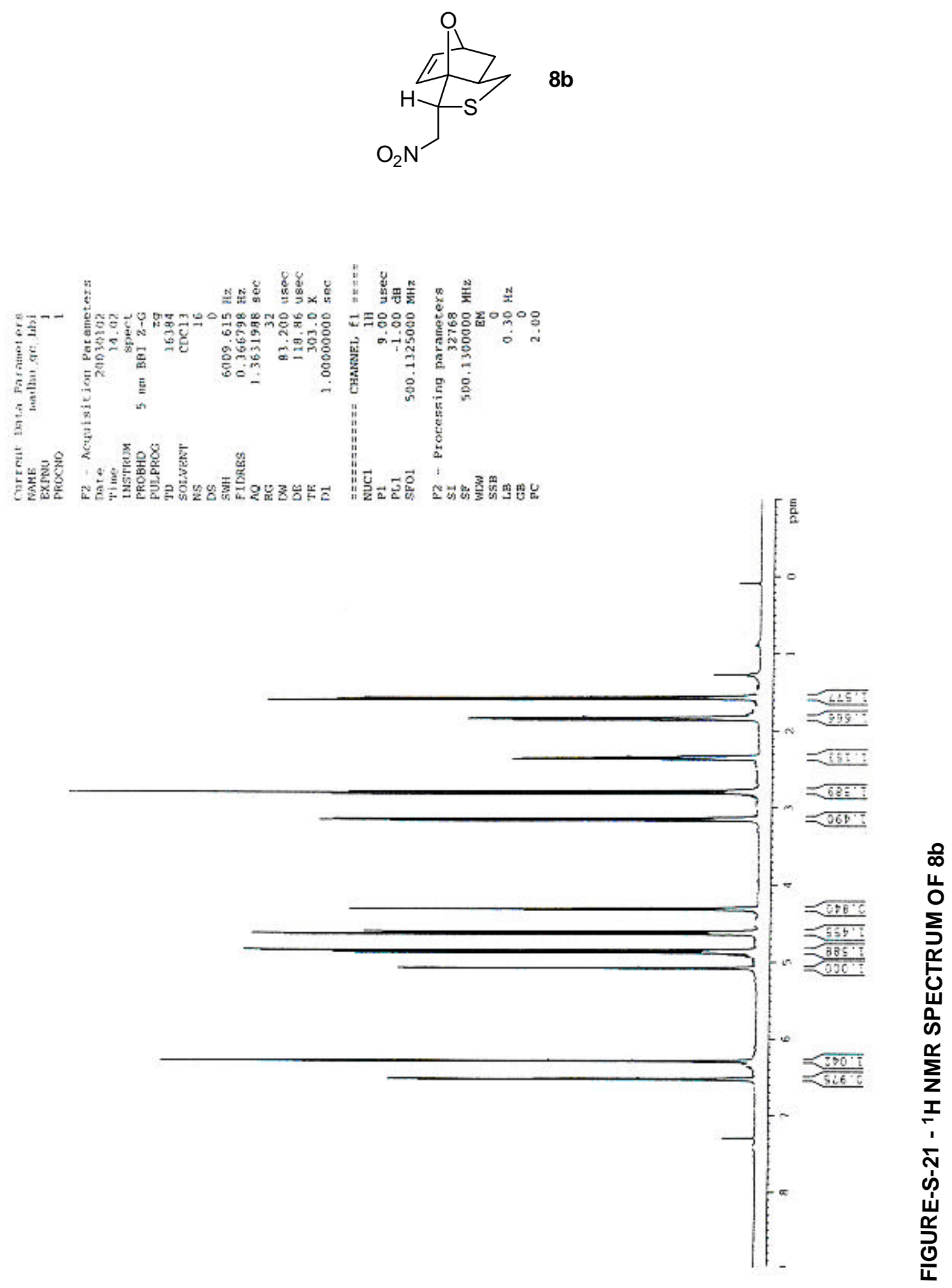


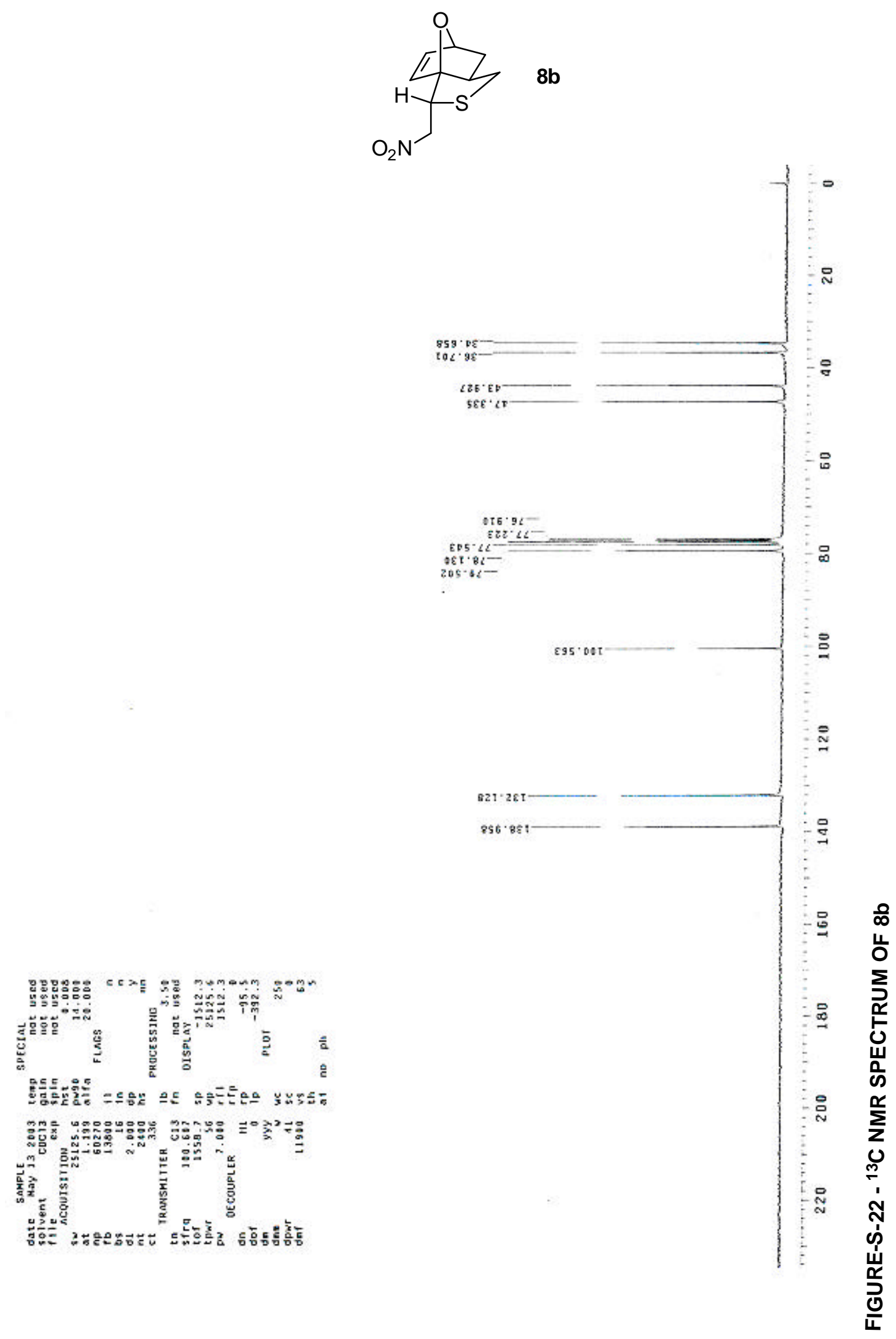



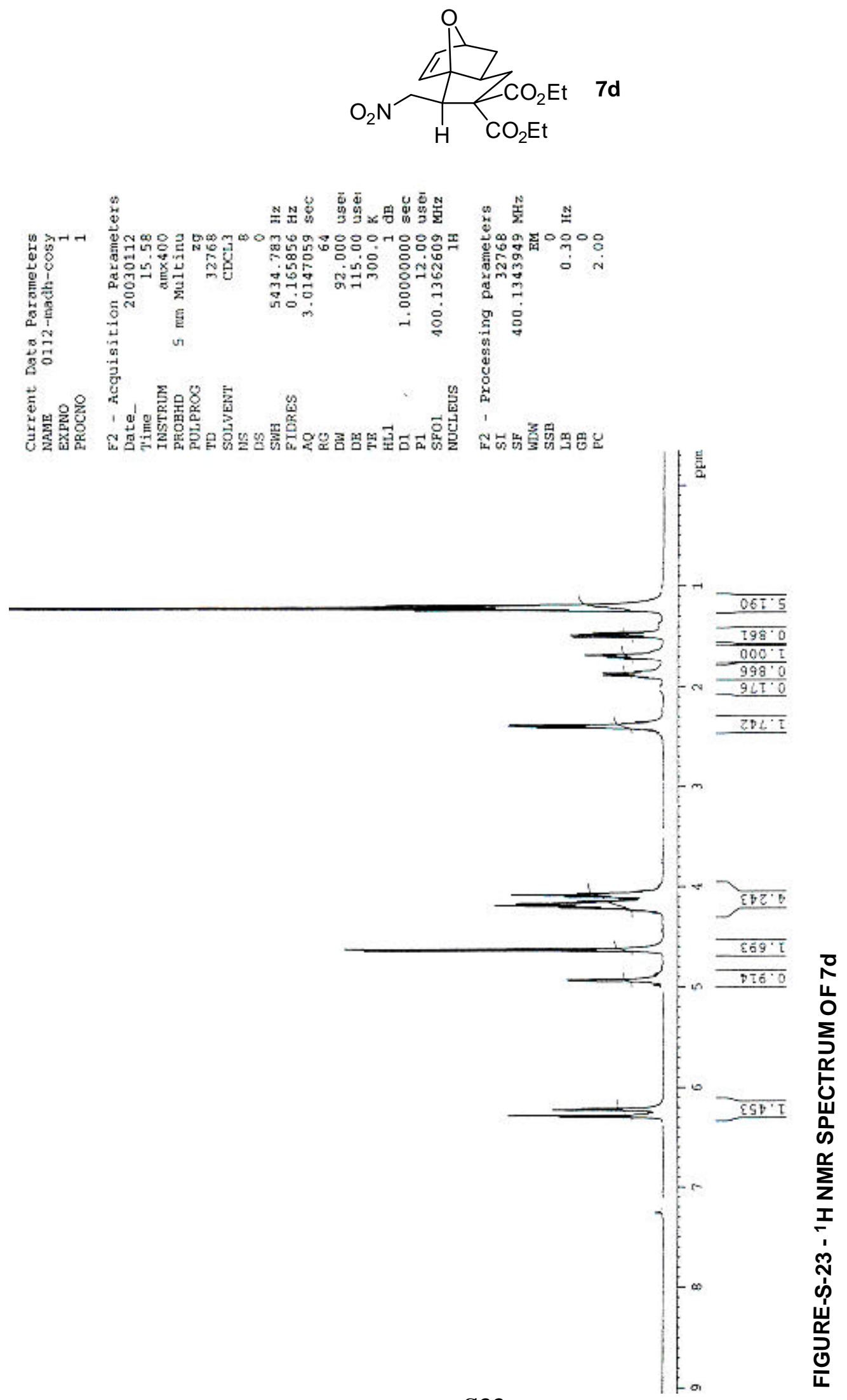

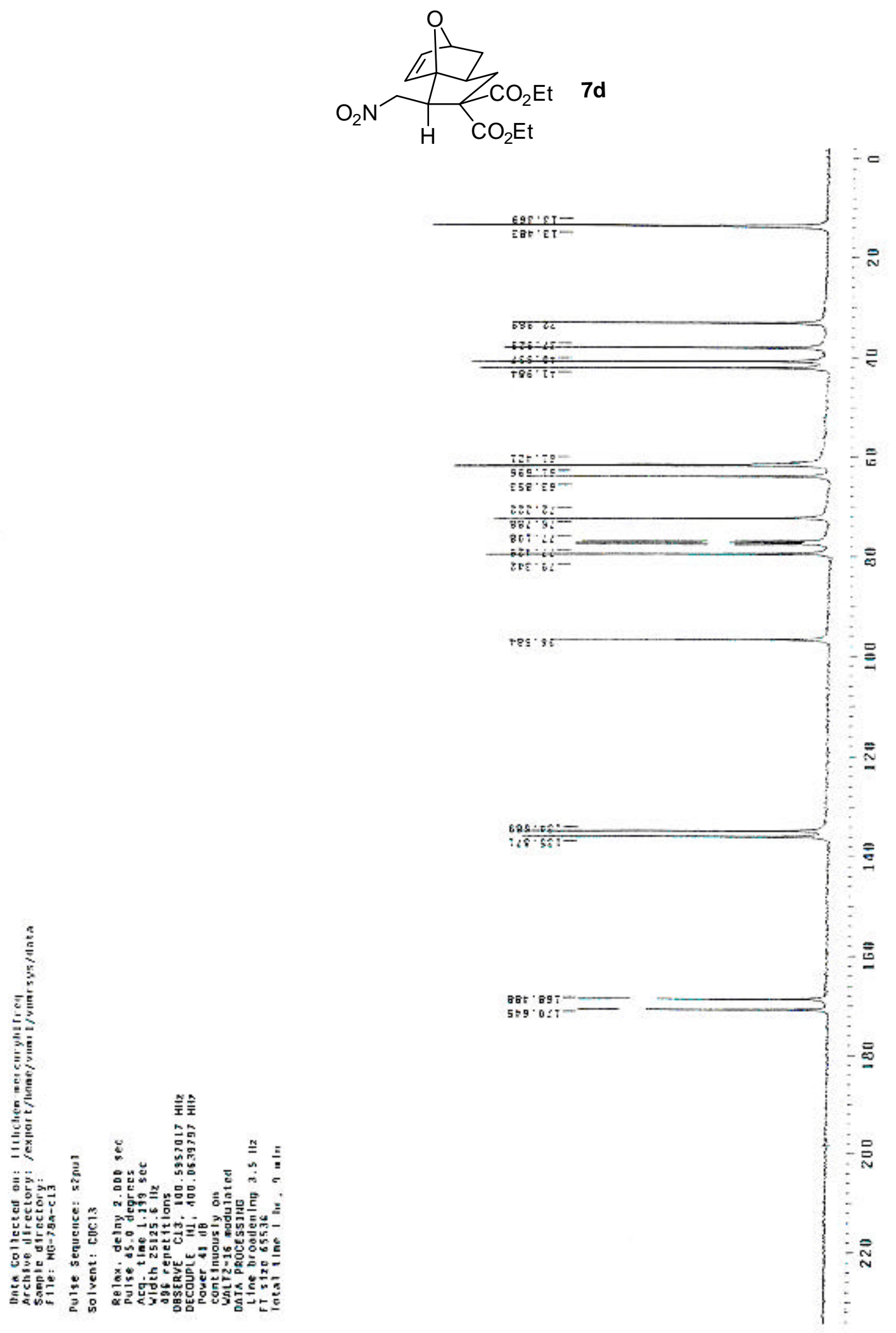

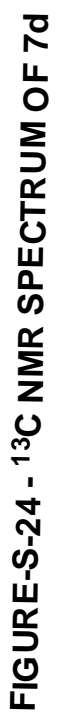




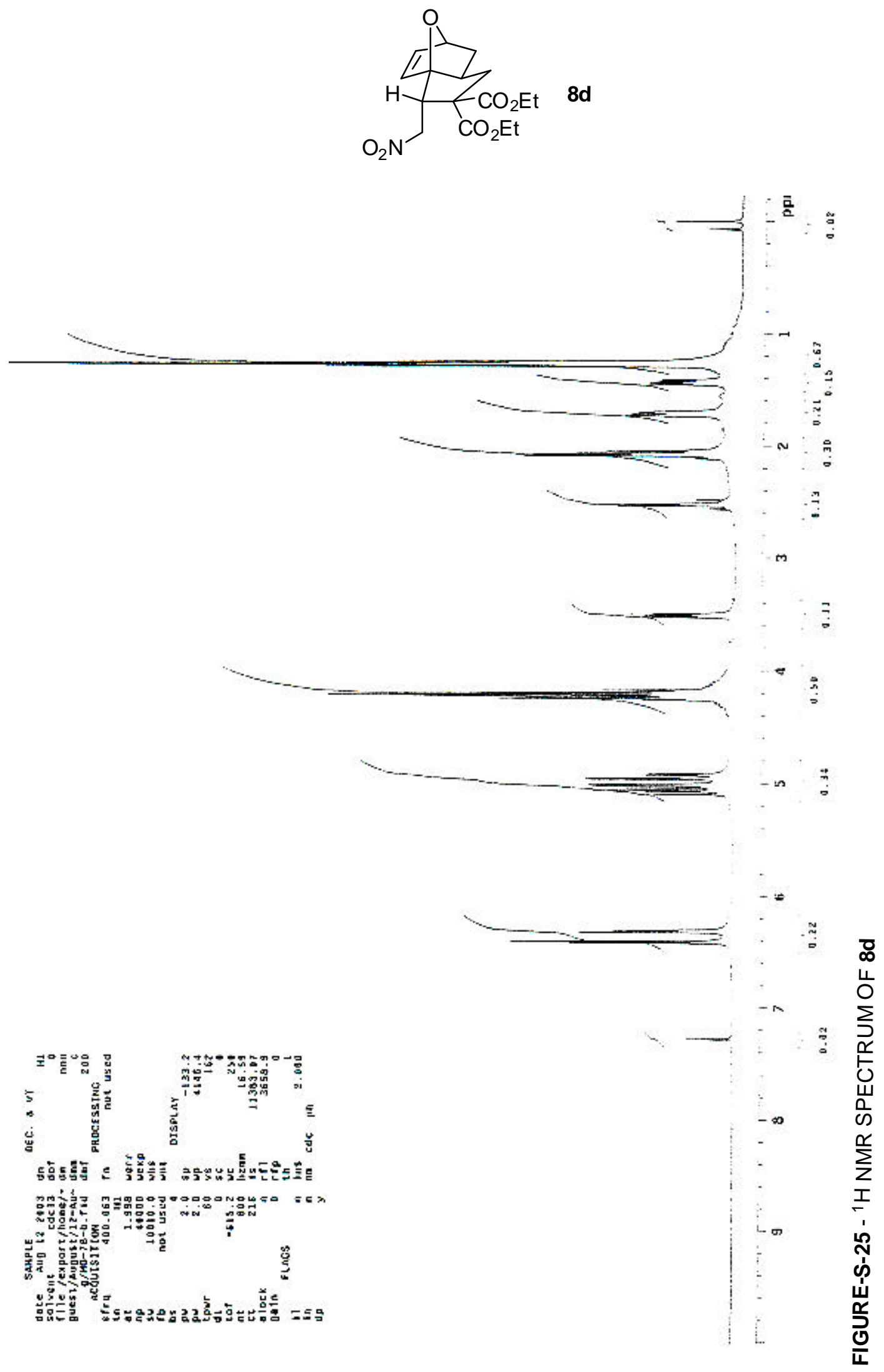

S35 


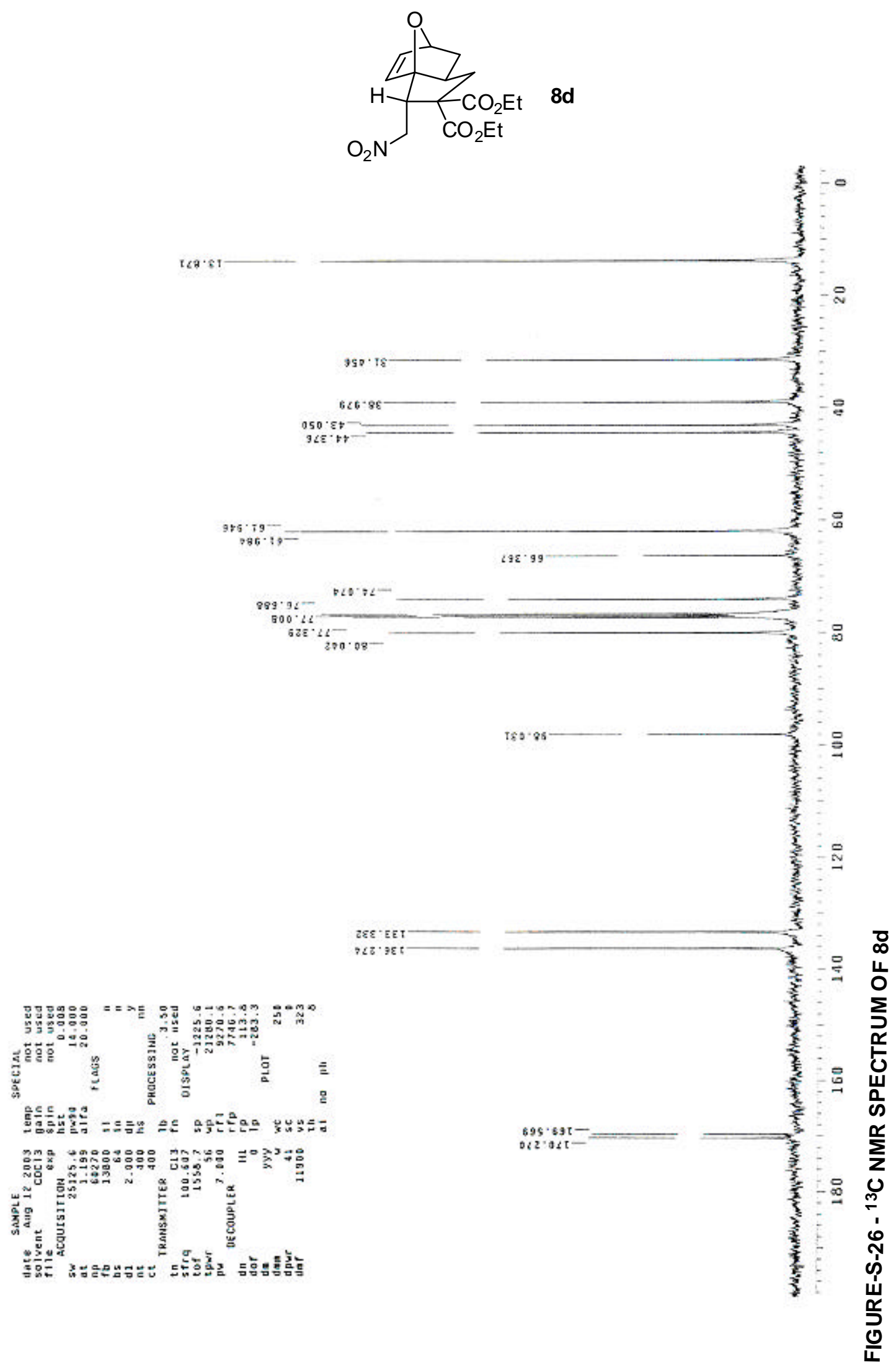




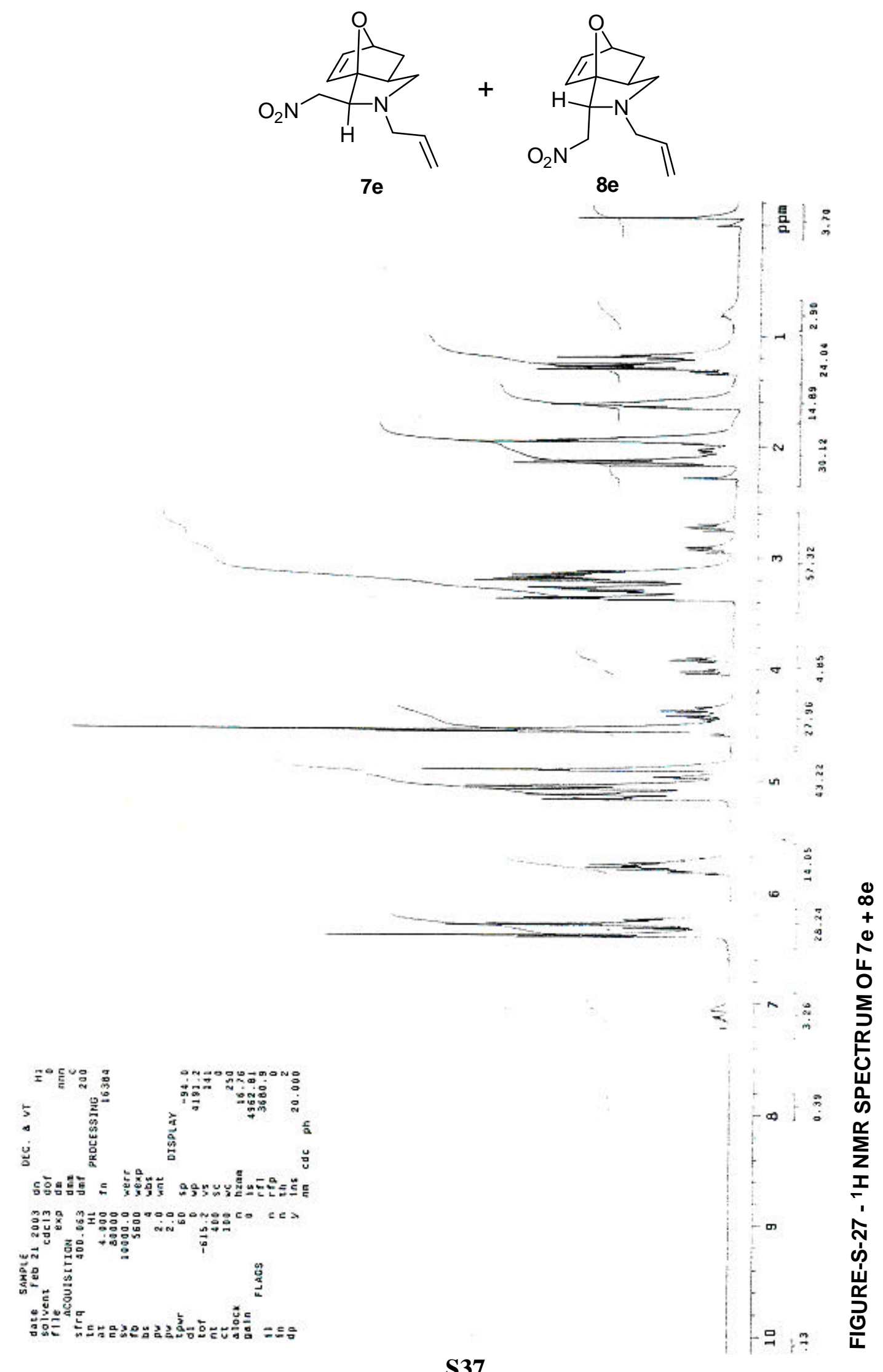




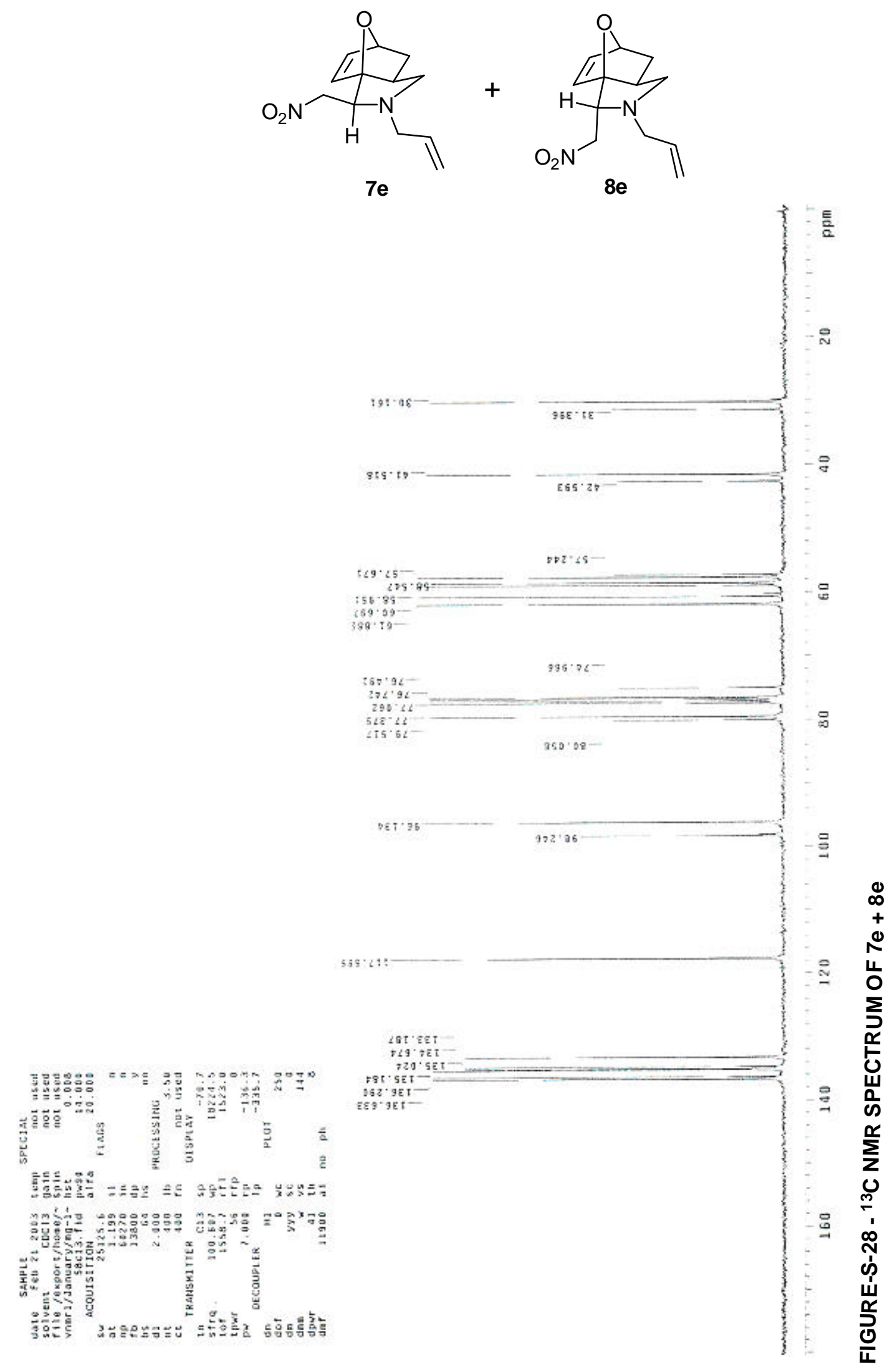




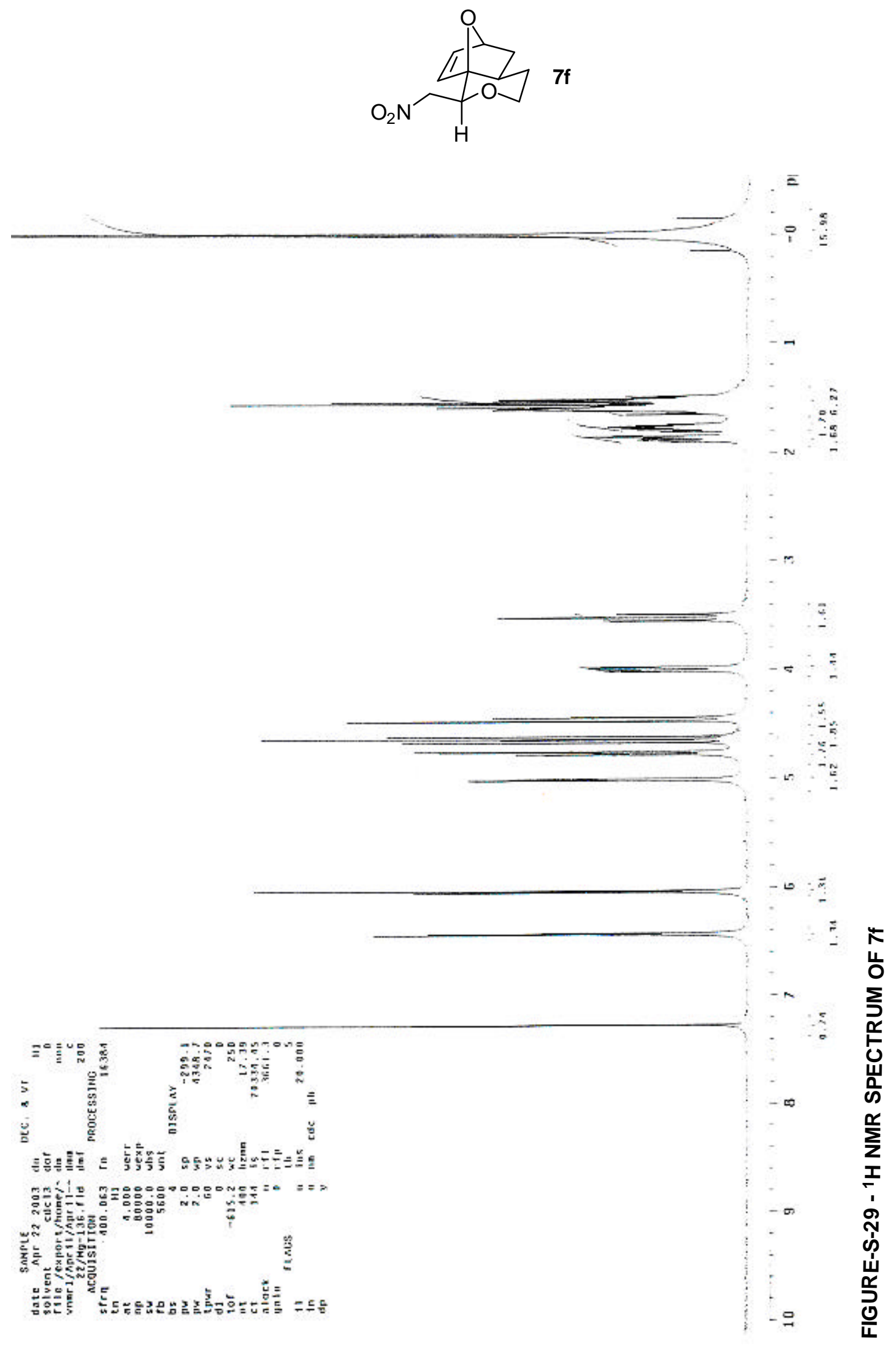




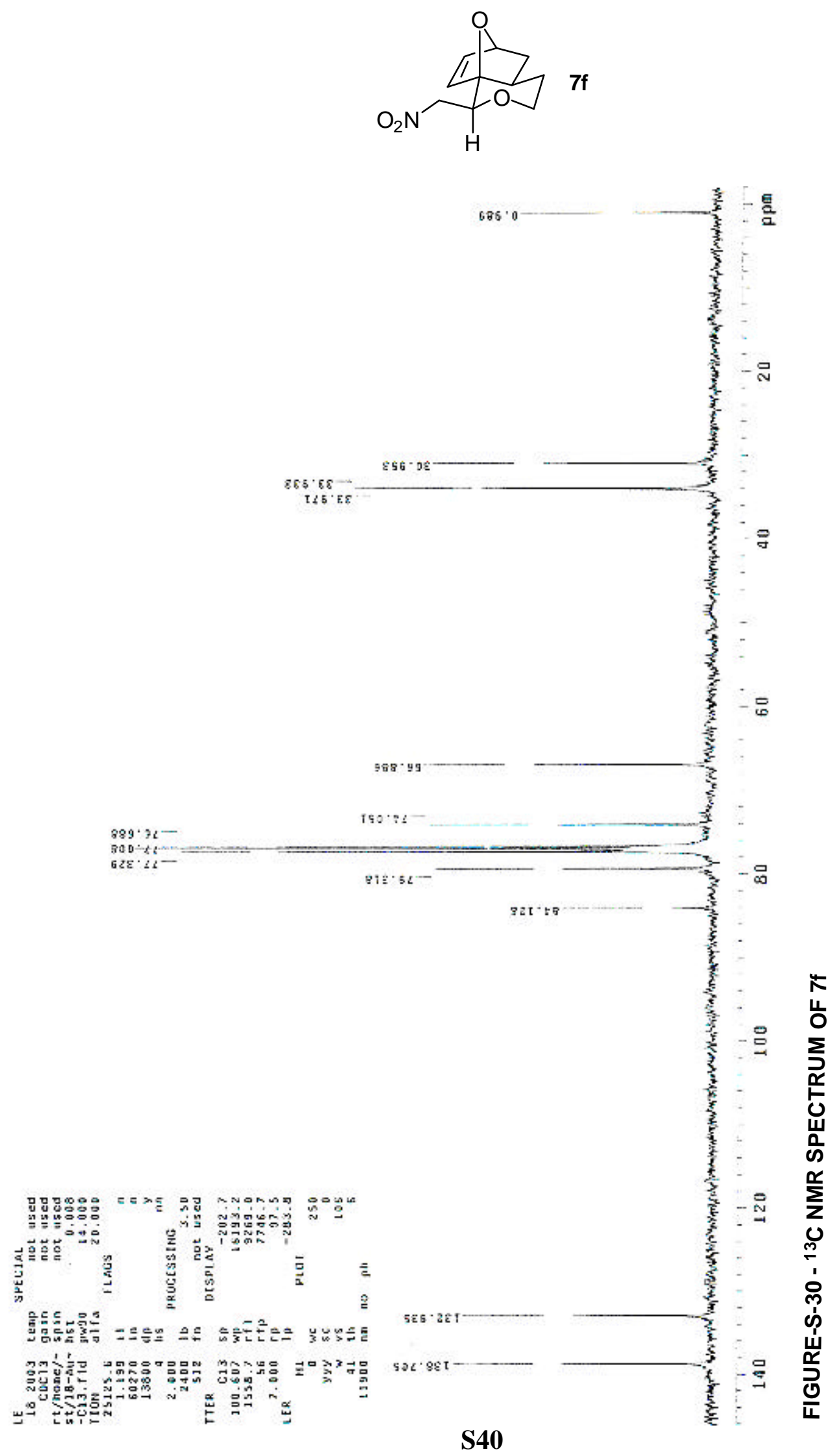




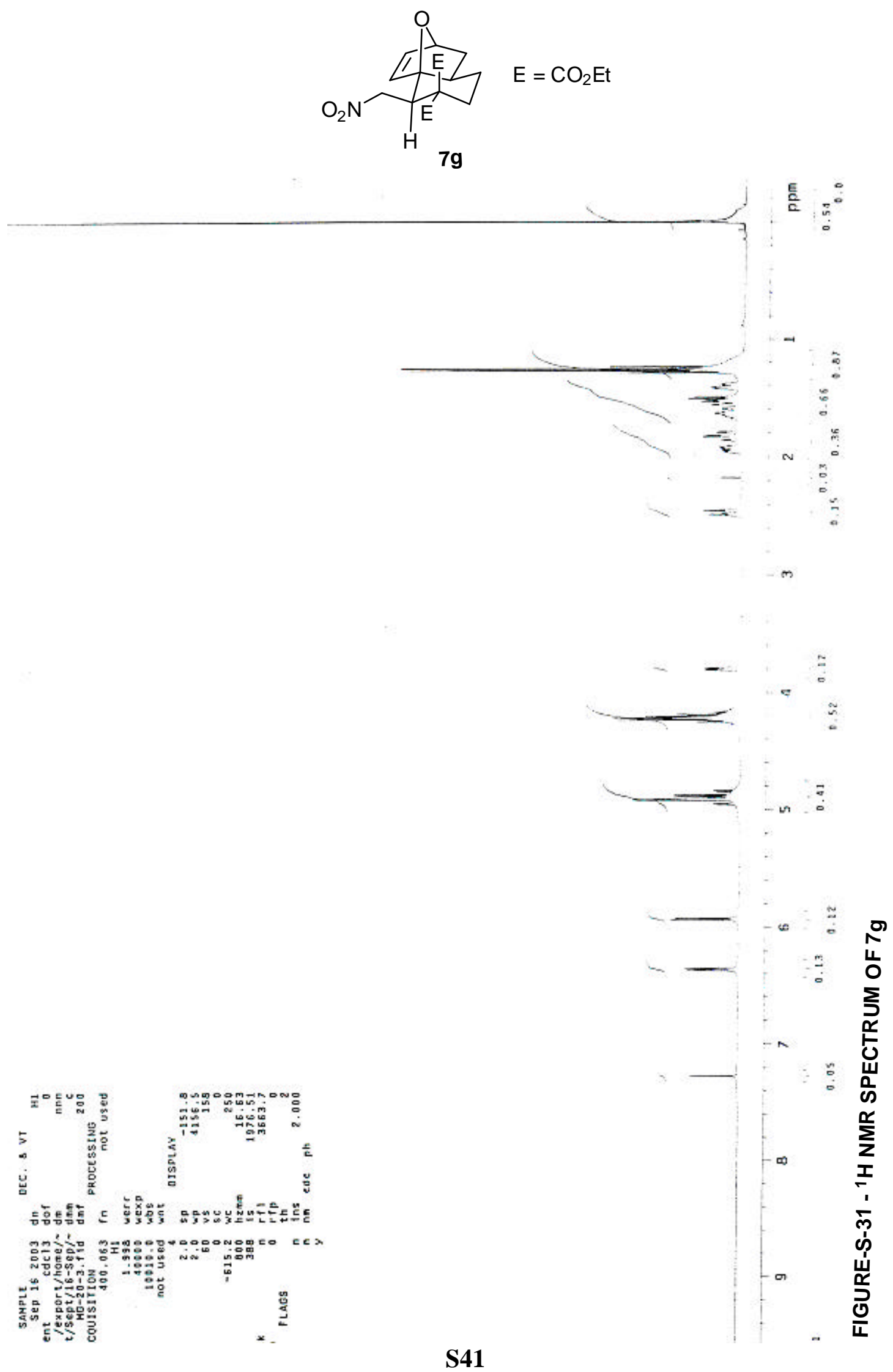




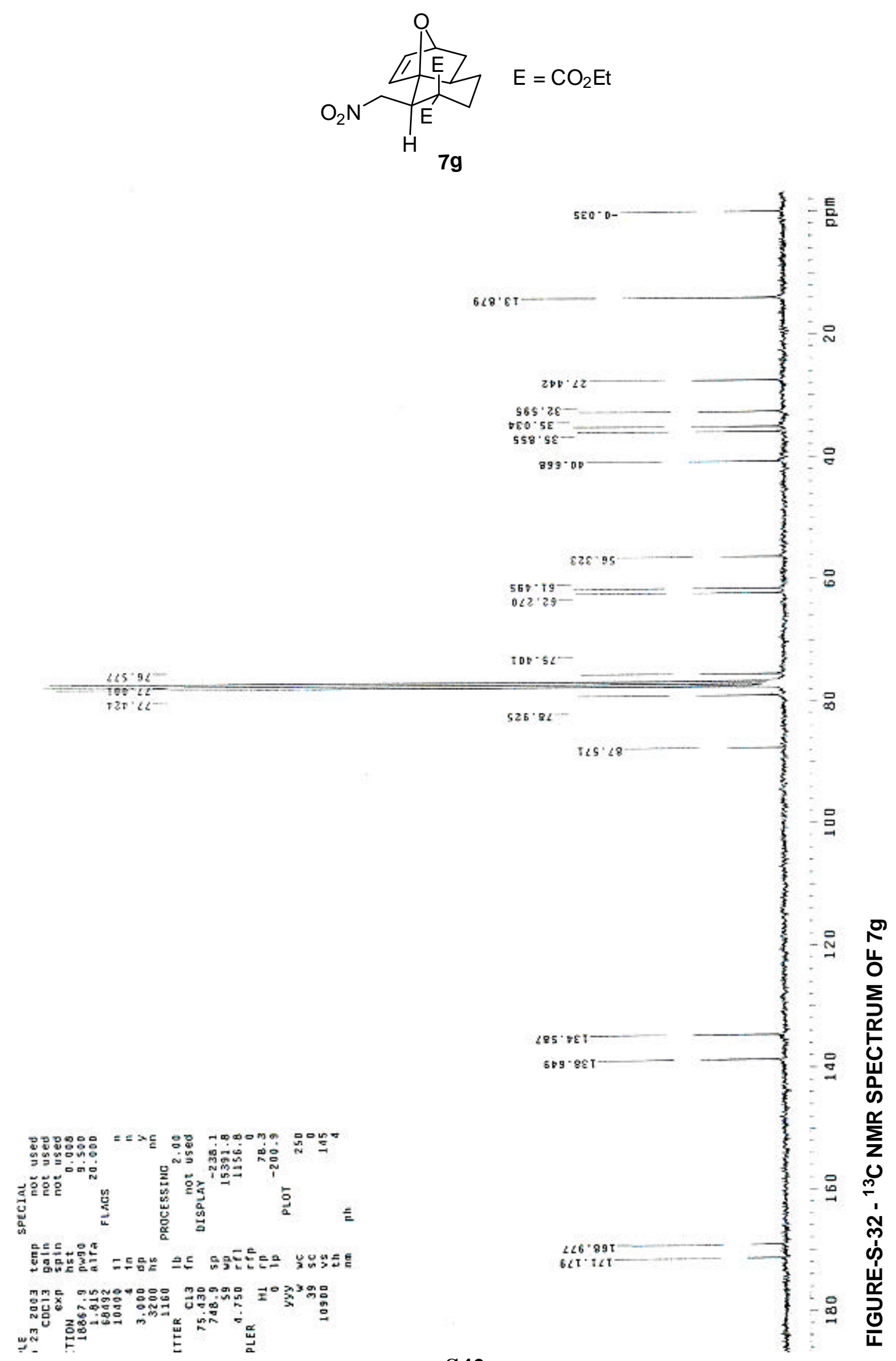




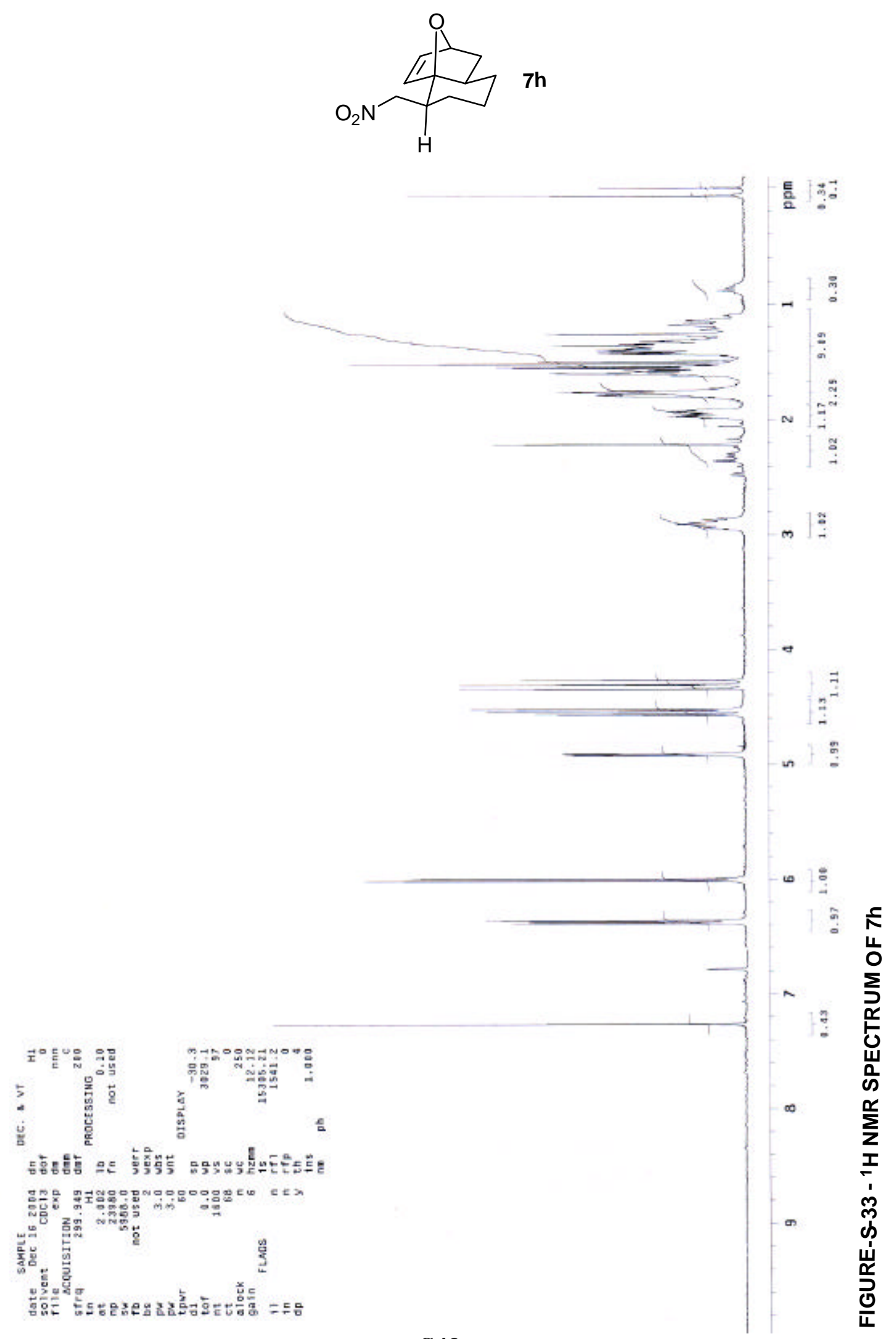




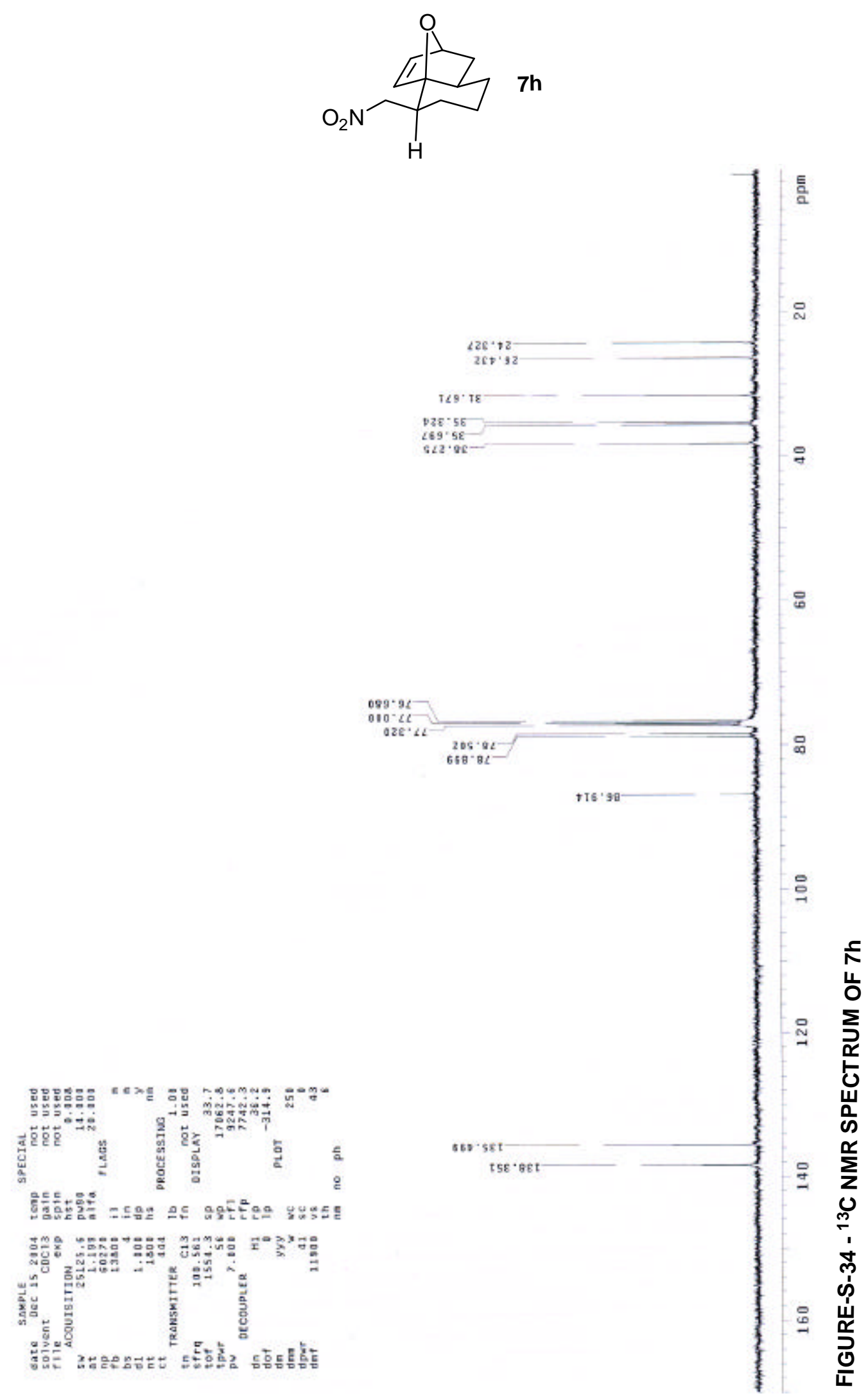




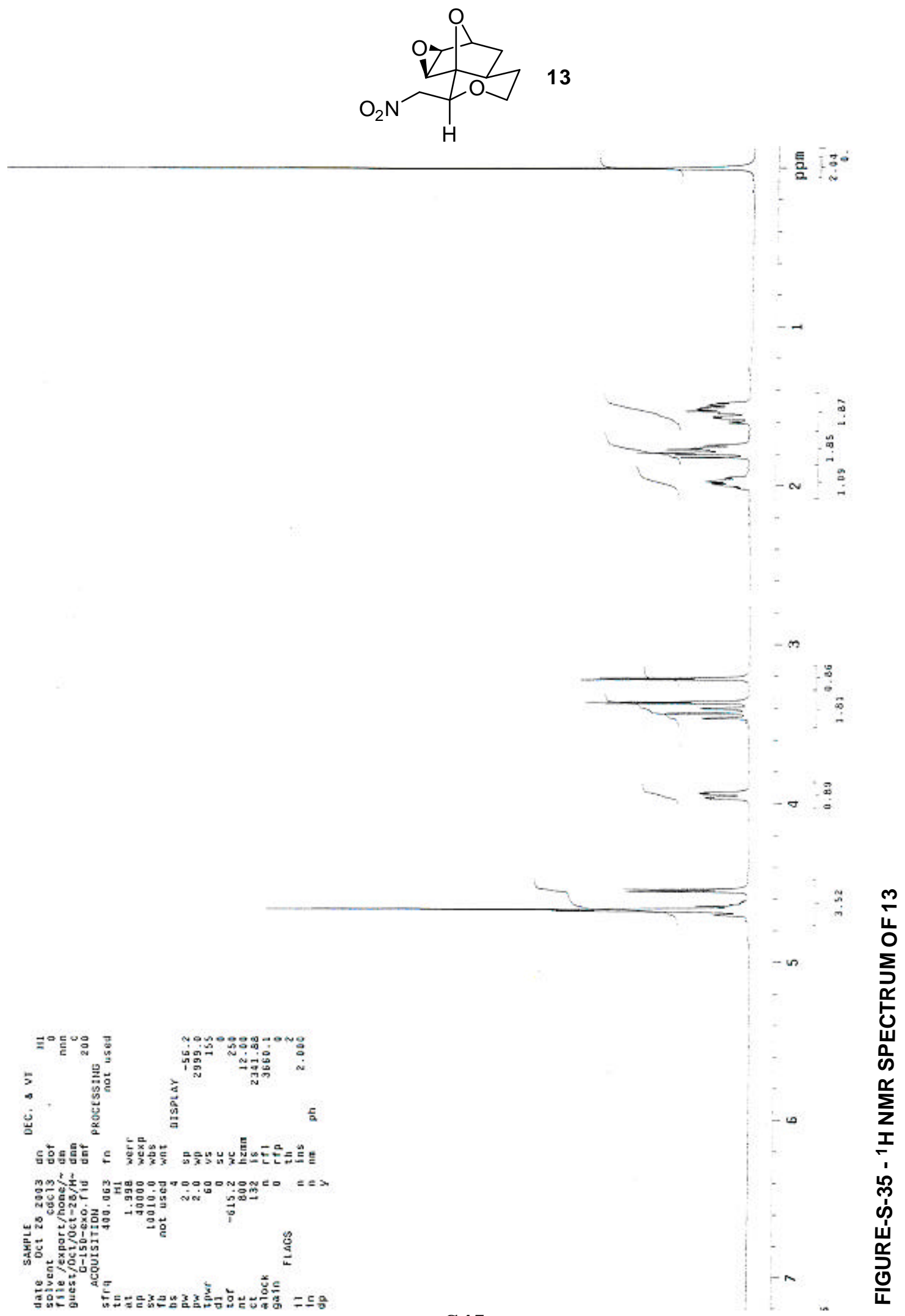




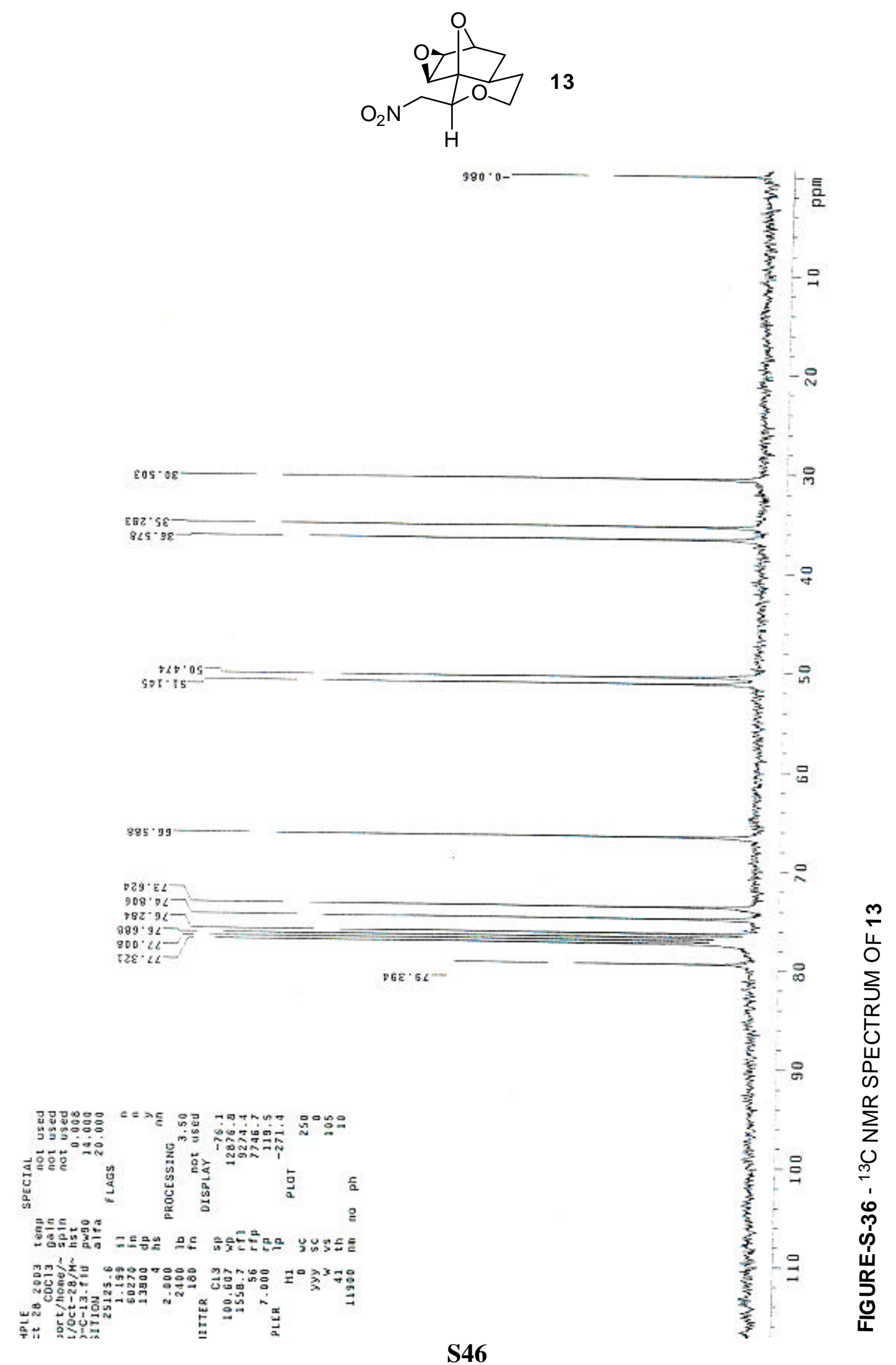




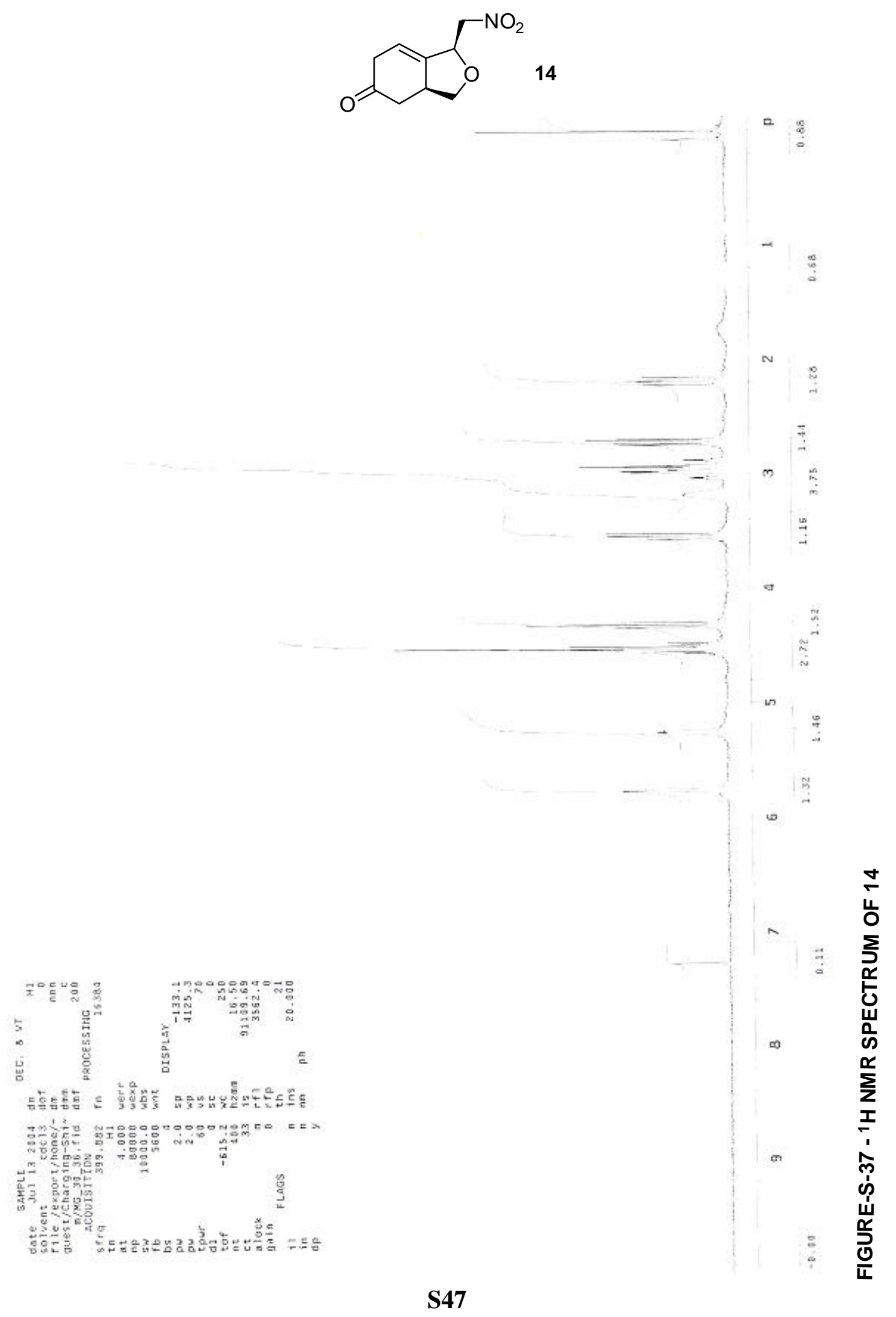




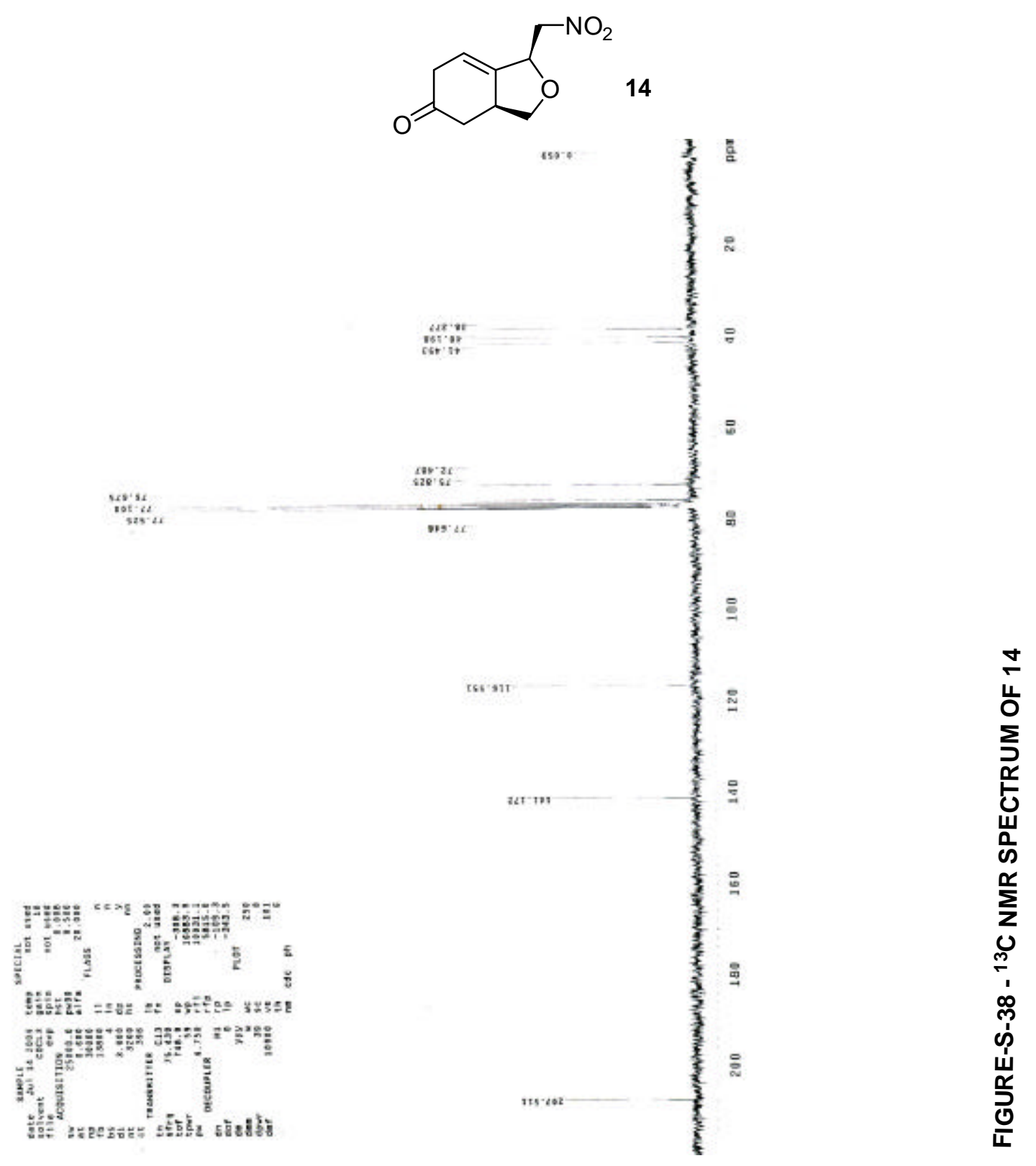




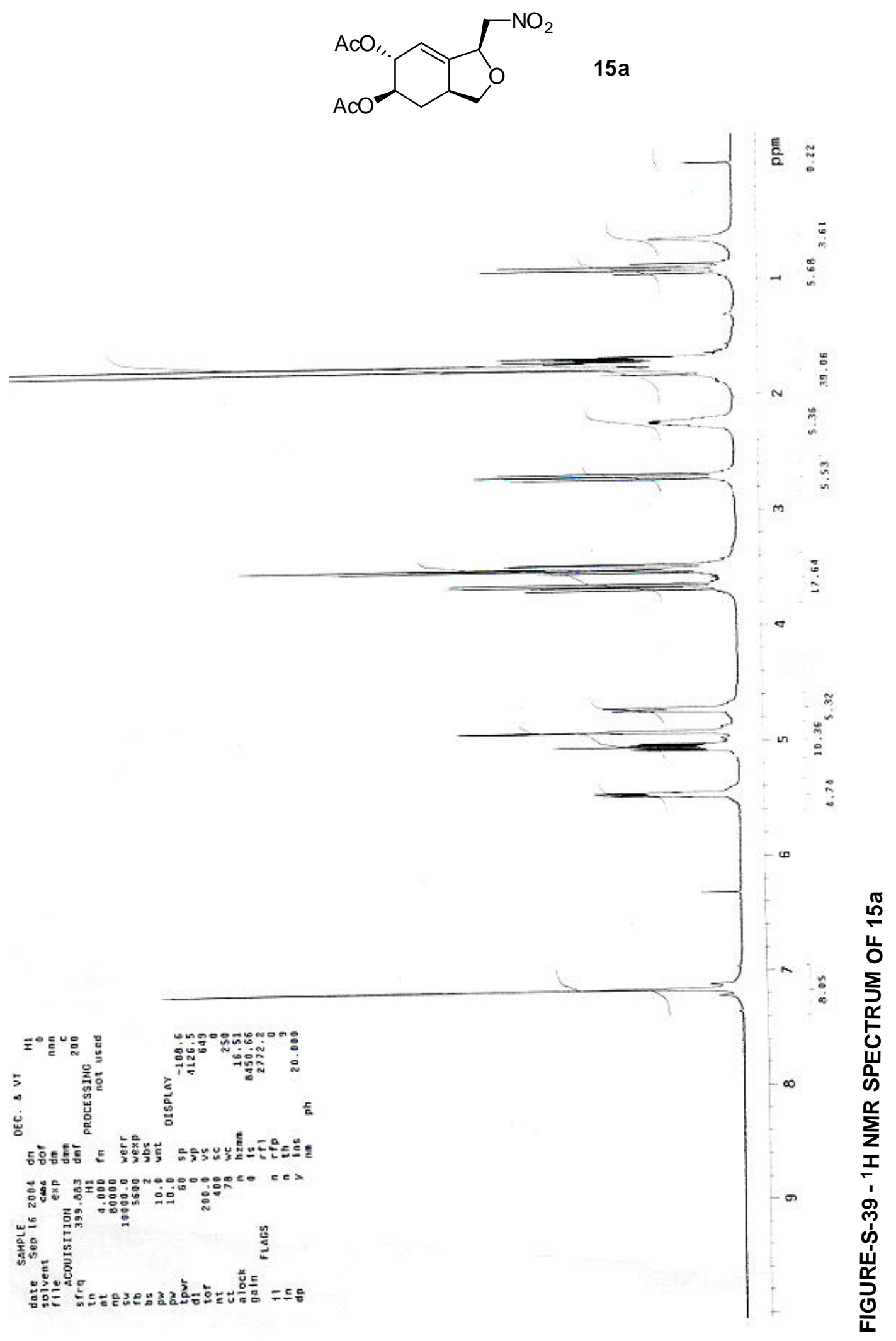




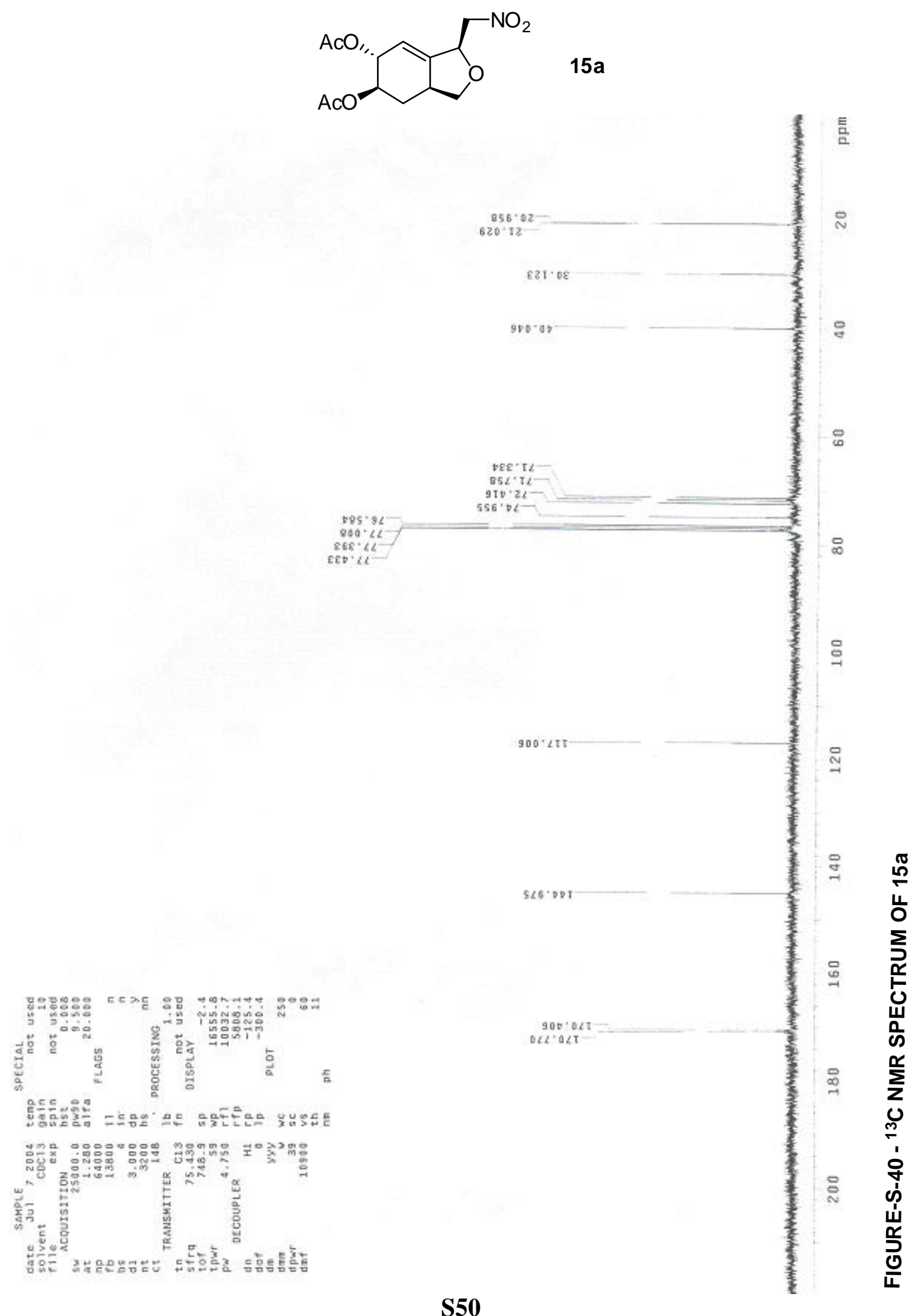



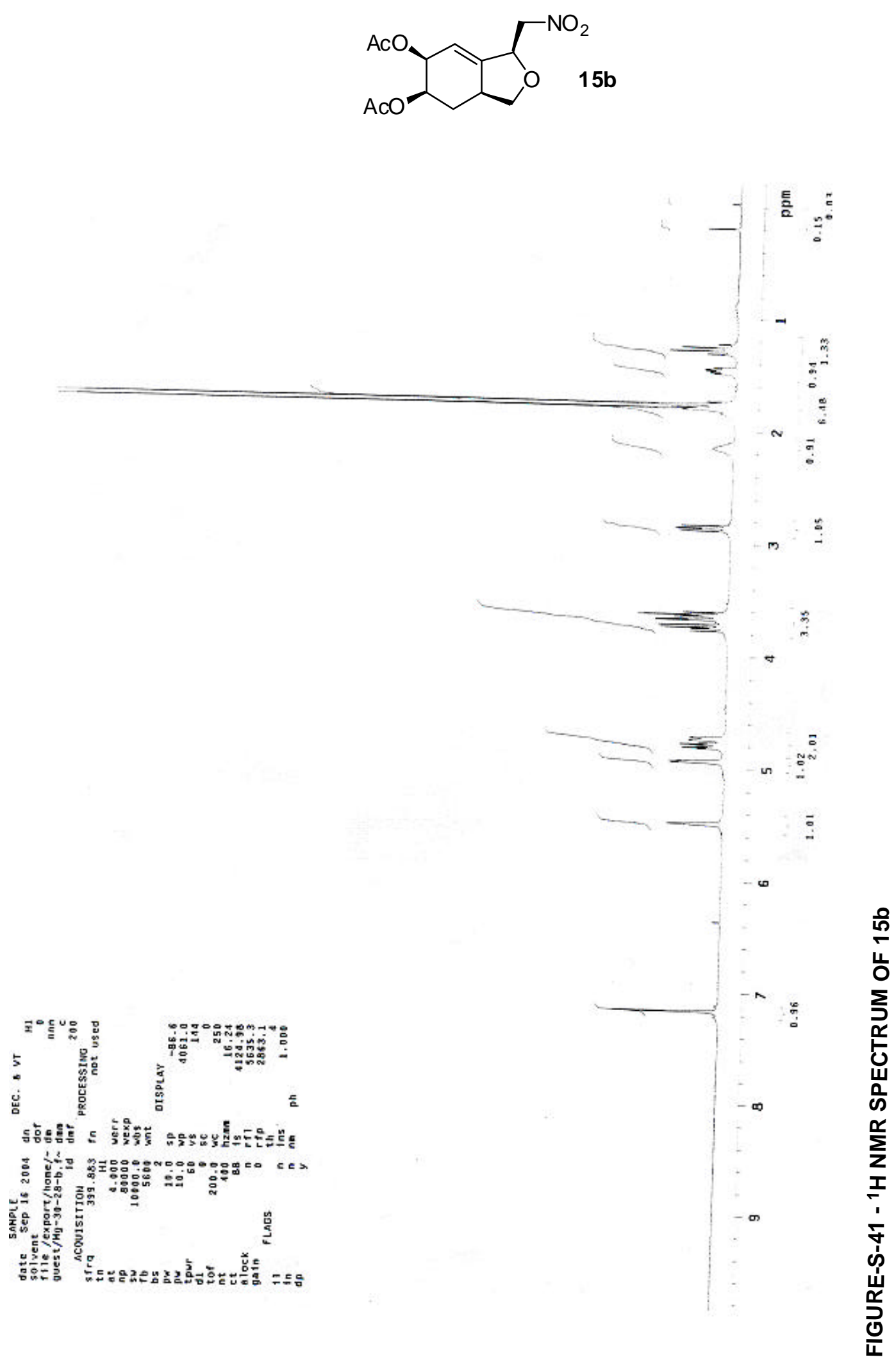


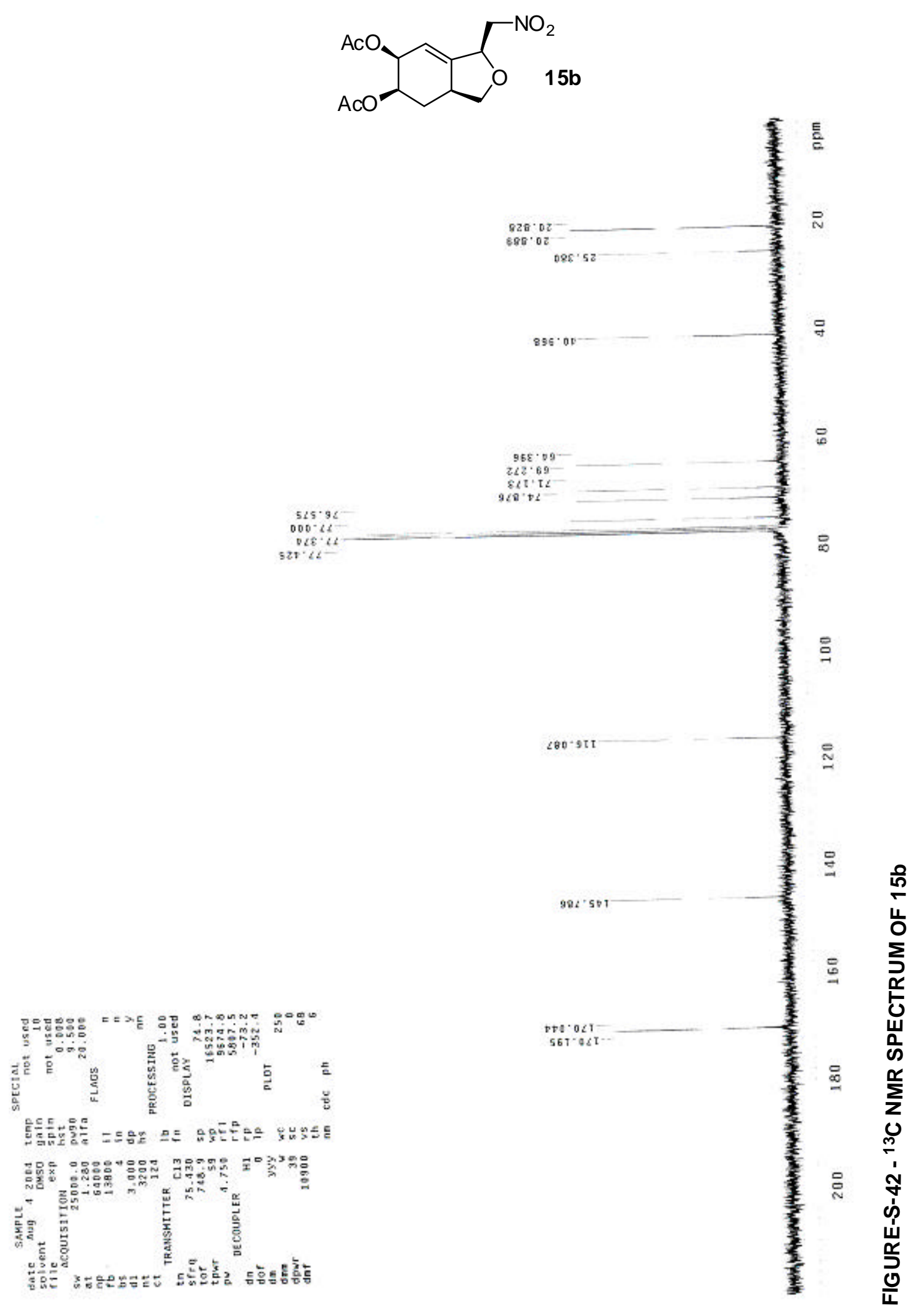


